\title{
FINITE VOLUME SCHEMES FOR THE BIHARMONIC PROBLEM ON GENERAL MESHES
}

\author{
R. EYMARD, T. GALLOUËT, R. HERBIN, AND A. LINKE
}

\begin{abstract}
During the development of a convergence theory for Nicolaides' extension of the classical MAC scheme for the incompressible Navier-Stokes equations to unstructured triangle meshes, it became clear that a convergence theory for a new kind of finite volume discretizations for the biharmonic problem would be a very useful tool in the convergence analysis of the generalized MAC scheme. Therefore, we present and analyze new finite volume schemes for the approximation of a biharmonic problem with Dirichlet boundary conditions on grids which satisfy an orthogonality condition. We prove that a piecewise constant approximate solution of the biharmonic problem converges in $L^{2}(\Omega)$ to the exact solution. Similar results are shown for the discrete approximate of the gradient and the discrete approximate of the Laplacian of the exact solution. Error estimates are also derived. This part of the paper is a first, significant step towards a convergence theory of Nicolaides' extension of the classical MAC scheme. Further, we show that finite volume discretizations for the biharmonic problem can also be defined on very general, nonconforming meshes, such that the same convergence results hold. The possibility to construct a converging lowest order finite volume method for the $H^{2}$-regular biharmonic problem on general meshes seems to be an interesting result for itself and clarifies the necessary ingredients for converging discretizations of the biharmonic problem. All these results are confirmed by numerical results.
\end{abstract}

\section{INTRODUCTION}

More than a hundred years ago, W. Ritz 27] computed the solutions of the biharmonic equation, in view of the study of the thin plate equilibrium. His pioneering idea was to introduce an approximate energy functional, replacing the exact solution by an expansion of smooth functions in the functional to be minimized, and leading to the resolution of a finite dimensional linear system. As mentioned in the very interesting historical article [18, this was probably the first brick to the foundation of the finite element method. Since then, a large number of discretization methods for the biharmonic operator have been proposed. The most classical is probably the conforming finite element method. For fourth order problems, the conforming finite element space must be a finite dimensional subspace of the Sobolev space $H^{2}(\Omega)$. Hence elementary basis functions are sought such that the reconstructed global basis functions on $\Omega$ belong to $C^{1}(\bar{\Omega})$. On Cartesian meshes, such basis functions are found by generalizing the one-dimensional $P^{3}$ Hermite finite element to the multi-dimensional framework. This task becomes much more difficult on more general meshes and involves rather sophisticated finite elements such as the

Received by the editor April 16, 2010 and, inrevised form, April 22, 2011.

2010 Mathematics Subject Classification. Primary 65N08.

Key words and phrases. Biharmonic problem, finite volume scheme, convergence analysis, error estimate. 
Argyris finite element on triangles in 2D, which unfortunately requires 21 degrees of freedom [8]. Hence nonconforming FEMs have also been widely studied; see e.g. [8, Section 49], 9], and references therein, and [4, 5] for more recent works. Discontinuous Galerkin methods have also been recently developed and analysed [20, 23, 28, 19]; error estimates have been derived for polynomials of degree greater or equal to two or three. Other methods which have been developed for fourth order problems include mixed methods [6] (see also references therein), 29], and compact finite difference methods [7, 3, 2,

Let us remark that the discretization of the biharmonic operator also becomes useful when considering the two-dimensional Navier-Stokes equations. Indeed, for any divergence-free function $\boldsymbol{v} \in\left[H_{0}^{1}(\Omega)\right]^{2}$, we can find a unique streamfunction $\psi \in H_{0}^{2}(\Omega)$ which fulfills $\boldsymbol{v}=\operatorname{curl} \psi$ and $\operatorname{rot} \boldsymbol{v}=\operatorname{rot} \operatorname{curl} \psi=-\Delta \psi$. Therefore, we can find for any smooth, divergence-free function $\boldsymbol{v}$ with compact support its vector potential by solving

$$
\Delta \Delta \psi=-\Delta \operatorname{rot} v
$$

In a recent paper [10, a discrete analogue of this relation is used to present a convergence analysis for Nicolaides' extension [21, 24] of the classical MAC scheme for the incompressible Navier-Stokes equations. This scheme is interesting because it is easy to implement, computationally cheap and robust, like the classical MAC scheme. In fact, using discrete stream functions it can be shown that the corresponding discretely divergence-free functions of the scheme possess an approximation property with respect to all divergence-free functions in $\left[H_{0}^{1}(\Omega)\right]^{2}$; see $[10$.

In Nicolaides' extension of the MAC scheme, unstructured triangular grids satisfying some orthogonality properties (Delaunay) [12] are applied. Therefore, biharmonic problems for this kind of meshes are investigated in the following. However, the discrete finite volume Laplace operator involved in the weak formulation for the biharmonic problem (on both solution and test function) is known to be nonconsistent in the finite difference sense on general unstructured meshes, i.e., when applied to the interpolation of a regular function [12, Section 5]; hence the usual finite difference convergence analysis technique fails. In the usual second order diffusion framework, this difficulty is circumvented by using the consistency of the normal diffusion flux [17]; in the weak formulation, this can be viewed as using the strong convergence of a discrete gradient of the interpolate of the test function [16.

In the case of the biharmonic operator, it is no longer a discrete gradient that we have to handle for a test function, but a discrete Laplacian. This is rather annoying, since, as we pointed out, the discrete Laplacian of a smooth test function will not, in general, converge strongly to the Laplacian of the test function. Therefore, the convergence technique used in the Laplace equation setting does not work; we deal with this new difficulty by a new interpolation procedure based on the solution of an auxiliary discrete problem (Lemmas 4.4 and 5.3 ).

The scheme which we propose consists of an approximation by piecewise constant functions of a weak formulation of the biharmonic problem. We address two types of meshes:

- we first deal with meshes which respect an adequate orthogonality property; these meshes allow for the simplest approximation of normal fluxes.

- we then generalize the analysis to general polygonal meshes.

In the latter case, although the diffusion fluxes may not be approximated by a two point formula, a cell centered scheme may still be defined and proved to be 
convergent, thanks to the reconstruction of a discrete gradient. This "SUCCES" scheme (Scheme Using Conservativity and Consistency Error Stabilization) was proved to be convergent for the approximation of the Laplace equation [15] and the $p$-Laplace equation [14. This is, to our knowledge, the first scheme for the discretization of the biharmonic problem on general polygonal meshes for which a convergence proof is proposed. Let us mention that it also applies on more general biharmonic problems, as stated in some remarks below.

The paper is organized as follows. The continuous problem is presented in Section 2. The finite volume discretizations which are under consideration in this paper are presented in Section 3 , which allow to introduce the scheme on admissible meshes in Section 4. The mathematical analysis of this scheme is detailed in Section 4.2. The extension of the scheme to general polygonal meshes is presented in Section 5 as well as the main lines of its mathematical analysis. Numerical results using various types of meshes for one-, two- or three-dimensional problems are provided in Section 6. Some perspectives concerning the derivation of schemes for the biharmonic problem by standard conforming finite element schemes are finally drawn in Section 7

\section{The Continuous PRoblem}

Throughout this paper,

$d \in \mathbb{N} \backslash\{0\}$ denotes the space dimension,

$\Omega$ is an open polygonal bounded and connected subset of $\mathbb{R}^{d}$, with Lipschitz-continuous boundary $\partial \Omega$,

and

$$
f \in L^{2}(\Omega), \quad \ell \in L^{2}(\Omega) \text { and } \boldsymbol{g} \in\left(L^{2}(\Omega)\right)^{d} .
$$

Under these assumptions, we consider the following problem:

$$
\begin{aligned}
& u \in H_{0}^{2}(\Omega), \forall v \in H_{0}^{2}(\Omega), \\
& \int_{\Omega} \Delta u(x) \Delta v(x) \mathrm{d} x=\int_{\Omega}(f(x) v(x)+\boldsymbol{g}(x) \cdot \nabla v(x)+\ell(x) \Delta v(x)) \mathrm{d} x,
\end{aligned}
$$

where $H_{0}^{2}(\Omega)$ is the closure of the set $C_{c}^{\infty}(\Omega)$ of infinitely continuously differentiable functions with compact support. We recall that under assumptions (11)-(2), Problem (3) has one and only one solution, thanks to the Riesz theorem and to the fact that $\|\Delta u\|_{L^{2}(\Omega)}$ is an equivalent norm to $\|u\|_{H^{2}(\Omega)}$ in $H_{0}^{2}(\Omega)$. Indeed, the Poincaré inequality

$$
\forall u \in H_{0}^{1}(\Omega),\|u\|_{L^{2}(\Omega)} \leq \operatorname{diam}(\Omega)\|\nabla u\|_{L^{2}(\Omega)^{d}}
$$

and

$$
\forall u \in H_{0}^{2}(\Omega),-\int_{\Omega} u \Delta u \mathrm{~d} x=\int_{\Omega} \nabla u \cdot \nabla u \mathrm{~d} x
$$

imply

$$
\forall u \in H_{0}^{2}(\Omega),\|\nabla u\|_{L^{2}(\Omega)^{d}} \leq \operatorname{diam}(\Omega)\|\Delta u\|_{L^{2}(\Omega)} .
$$


Besides, the following equality which is an immediate consequence of two integrations by parts

$$
\begin{aligned}
\forall \varphi \in C_{c}^{\infty}(\Omega), \int_{\Omega}(\Delta \varphi(x))^{2} \mathrm{~d} x & =\sum_{i=1}^{d} \sum_{j=1}^{d} \int_{\Omega} \partial_{i i}^{2} \varphi(x) \partial_{j j}^{2} \varphi(x) \mathrm{d} x \\
& =\sum_{i=1}^{d} \sum_{j=1}^{d} \int_{\Omega}\left(\partial_{i j}^{2} \varphi(x)\right)^{2} \mathrm{~d} x,
\end{aligned}
$$

completes the proof of the equivalence of the norms.

\section{Finite volume meshes}

Roughly speaking, a finite volume mesh is a partition of $\Omega$ into polygonal or polyhedral subsets. In Section 4 below, we first consider the $\Delta$-adapted (admissible meshes of [12, Definition 9.1]), for which the diffusion flux $\nabla u \cdot \boldsymbol{n}$ may be approximated by a consistent two point formula. In Section 5 we treat the case of the general meshes of [15, Definition 2.1], thanks to the machinery of this latter reference. For the sake of clarity and concision, we introduce in the present section the common features of both meshes. Since we are addressing higher order differential operators, the fluxes to be discretized involve higher order derivatives, so that the definition of the flux consistency is based on some points in the discretization cells. Let us first describe the general finite volume (FV) meshes, which are depicted in Figure 1

Definition 3.1 (General FV discretization). Under hypothesis (11), a general FV discretization of $\Omega$, is given by the triplet $\mathcal{D}=(\mathcal{M}, \mathcal{E}, \mathcal{P})$, where:

(1) $\mathcal{M}$ is a finite family of nonempty connected open disjoint subsets of $\Omega$ (the "control volumes") such that $\bar{\Omega}=\bigcup_{K \in \mathcal{M}} \bar{K}$. For any $K \in \mathcal{M}$, let $\partial K=\bar{K} \backslash K$ be the boundary of $K,|K|>0$ denote the measure of $K$ and $h_{K}$ denote the diameter of $K$, that is, the maximum distance between two points of $K$.

(2) $\mathcal{E}=\mathcal{E}_{\text {int }} \cup \mathcal{E}_{\text {ext }}$ is a finite family of disjoint subsets of $\bar{\Omega}$ (the "interfaces" of the mesh, i.e., the edges in $2 \mathrm{D}$ and faces in $3 \mathrm{D}$ ), such that, for all $\sigma \in \mathcal{E}_{\text {int }}$, $\sigma$ is a nonempty open subset of a hyperplane of $\mathbb{R}^{d}$ included in $\Omega$ and for all $\sigma \in \mathcal{E}_{\text {ext }}, \sigma$ is a nonempty open subset of $\partial \Omega$; furthermore, the $(d-1)$ dimensional measure $|\sigma|$ of any $\sigma \in \mathcal{E}$ is strictly positive. We assume that, for all $K \in \mathcal{M}$, there exists a subset $\mathcal{E}_{K}$ of $\mathcal{E}$ such that $\partial K=\bigcup_{\sigma \in \mathcal{E}_{K}} \bar{\sigma}$. We then denote by $\mathcal{M}_{\sigma}=\left\{K \in \mathcal{M}, \sigma \in \mathcal{E}_{K}\right\}$. We then assume that, for all $\sigma \in \mathcal{E}$, either $\mathcal{M}_{\sigma}$ has exactly one element and then $\sigma \in \mathcal{E}_{\text {ext }}$ or $\mathcal{M}_{\sigma}$ has exactly two elements and then $\sigma \in \mathcal{E}_{\text {int }}$. For all $K \in \mathcal{M}$ and for any hyperplanar $\sigma \in \mathcal{E}_{K}$, we denote for a.e. $x \in \sigma$ by $\boldsymbol{n}_{K, \sigma}$ the (constant) unit vector normal to $\sigma$ outward to $K$.

(3) $\mathcal{P}$ is a family of points of $\Omega$ indexed by $\mathcal{M}$ and $\mathcal{E}$, denoted by $\mathcal{P}=$ $\left(\left(x_{K}\right)_{K \in \mathcal{M}},\left(x_{\sigma}\right)_{\sigma \in \mathcal{E}}\right)$, such that for all $K \in \mathcal{M}, x_{K} \in K$ and for all $\sigma \in \mathcal{E}$, $x_{\sigma}$ is the center of gravity of $\sigma$. We then denote by $d_{K, \sigma}$ the orthogonal distance between $x_{K}$ and $\sigma$. The family $\mathcal{P}$ is chosen so that all the cells $K \in \mathcal{M}$ are strictly $x_{K}$-star-shaped, that is, for all $x \in K$, the line segment 
$\left[x_{K}, x\right]$ is strictly included in $K$, or equivalently, $d_{K, \sigma} \geq 0$ for all $\sigma \in \mathcal{E}_{K}$. In particular, this star-shaped condition ensures that

$$
\sum_{\sigma \in \mathcal{E}_{K}}|\sigma| d_{K, \sigma}=d|K|, \forall K \in \mathcal{M}
$$

The size of the discretization is defined by:

$$
h_{\mathcal{D}}=\sup \left\{h_{K}, K \in \mathcal{M}\right\} .
$$

For all $K \in \mathcal{M}$ and $\sigma \in \mathcal{E}_{K}$, we denote by $D_{K, \sigma}$ the cone with vertex $x_{K}$ and basis $\sigma$

$$
D_{K, \sigma}=\left\{t x_{K}+(1-t) y, t \in(0,1), y \in \sigma\right\},
$$

We denote, for all $\sigma \in \mathcal{E}, D_{\sigma}=\bigcup_{K \in \mathcal{M}_{\sigma}} D_{K, \sigma}$ (this set is also called the "diamond cell" associated to the interface $\sigma$ ).

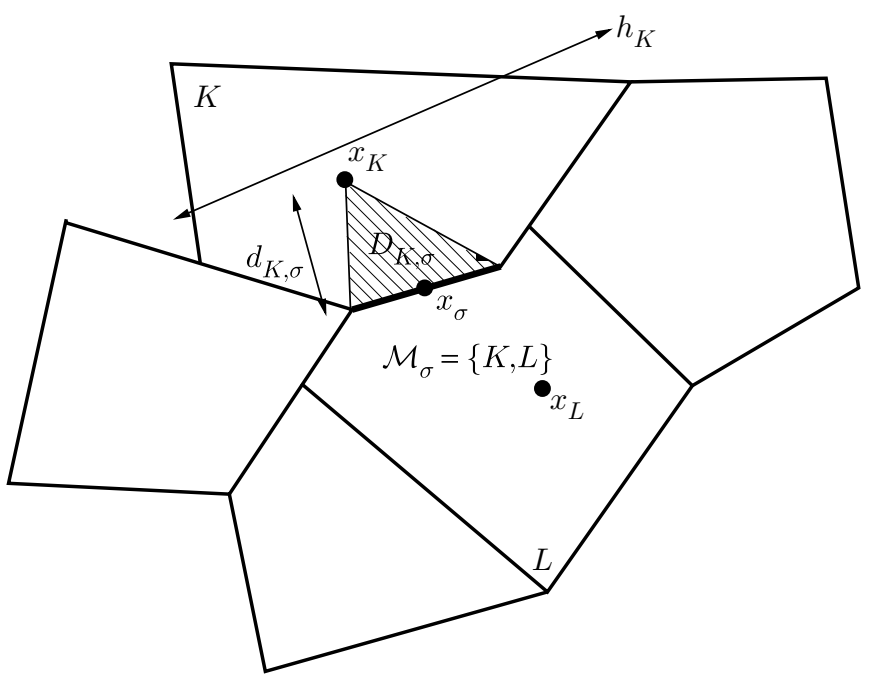

Figure 1. Notations for a control volume $K$ in the case $d=2$

Remark 3.1. The above definition applies to a large variety of meshes. In particular, control volumes are not assumed to be convex. Hence generalized "hexahedra" with nonplanar faces can be used (in fact, such sets then have 12 faces if each nonplanar face is shared in two triangles, but only 6 neighbouring control volumes). Note also that the common boundary of two neighbouring control volumes can include more than one interface.

Let us now introduce the notion of $\Delta$-adapted discretization, which are particular cases of general FV discretization, as depicted in Figure 2 for the two-dimensional case (recall that the schemes and their analysis are valid in both 2 and 3 dimensions).

Definition 3.2 ( $\Delta$-adapted FV discretization). Under hypothesis (1), a general $\mathrm{FV}$ discretization $\mathcal{D}=(\mathcal{M}, \mathcal{E}, \mathcal{P})$ in the sense of Definition 3.1 is $\Delta$-adapted if, for all $\sigma \in \mathcal{E}_{\text {int }}$, denoting by $K, L \in \mathcal{M}$ the two control volumes such that $\mathcal{M}_{\sigma}=\{K, L\}$, 


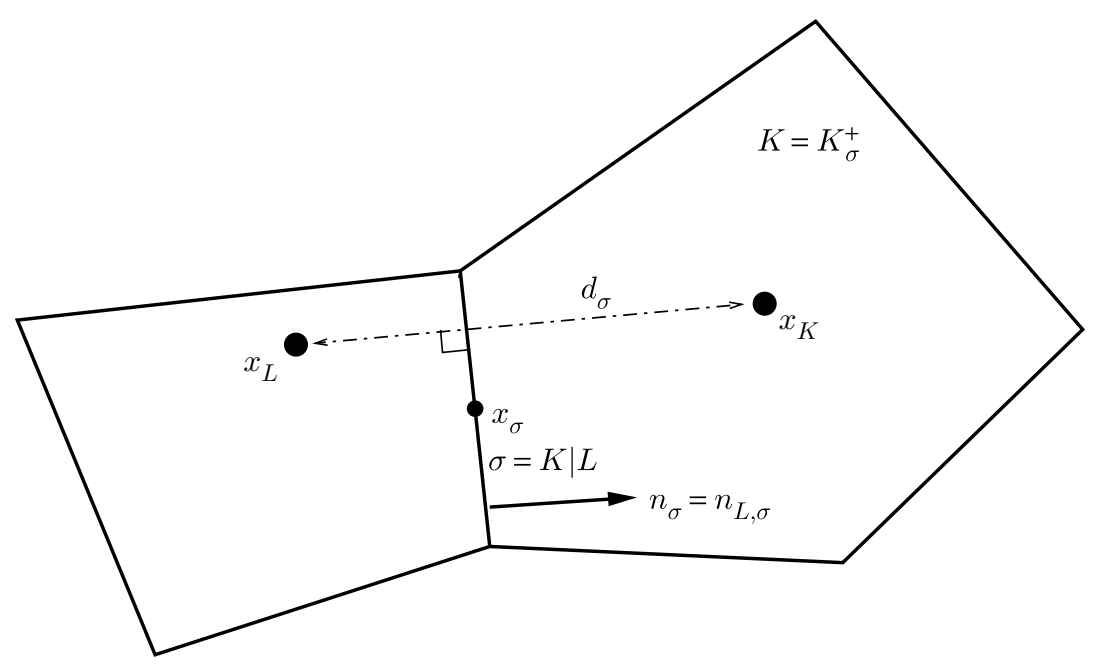

FIgURE 2. Notations for $\Delta$-adapted discretization

then the straight line $\left(x_{K}, x_{L}\right)$ is orthogonal to $\sigma$. Then, for $K, L \in \mathcal{M}$, such that there exists $\sigma \in \mathcal{E}_{\text {int }}$ with $\mathcal{M}_{\sigma}=\{K, L\}$, we assume that $\sigma$ is unique and one denotes $\sigma=K \mid L$. For all $\sigma \in \mathcal{E}_{\text {int }}$, an orientation is chosen by defining one of the two unit normal vectors $\boldsymbol{n}_{\sigma}$, for each $\sigma \in \mathcal{E}_{\text {int }}$, and we denote by $K_{\sigma}^{-}$and $K_{\sigma}^{+}$the two adjacent control volumes such that $\boldsymbol{n}_{\sigma}$ is oriented from $K_{\sigma}^{-}$to $K_{\sigma}^{+}$. We then set

$$
d_{\sigma}=\mathrm{d}\left(x_{K_{\sigma}^{-}}, x_{K_{\sigma}^{+}}\right)=\mathrm{d}\left(x_{K_{\sigma}^{-}}, \sigma\right)+\mathrm{d}\left(x_{K_{\sigma}^{+}}, \sigma\right) .
$$

For all $\sigma \in \mathcal{E}_{\text {ext }}$, we denote the control volume $K \in \mathcal{M}$ such that $\sigma \in \mathcal{E}_{K}$ by $K_{\sigma}$; we define

$$
d_{\sigma}=\mathrm{d}\left(x_{K_{\sigma}}, \sigma\right)
$$

and we define $\boldsymbol{n}_{\sigma}$ by $\boldsymbol{n}_{\sigma}=\boldsymbol{n}_{K_{\sigma}, \sigma}$. We define, for all $K \in \mathcal{M}, \mathcal{E}_{K \text {,int }}=\mathcal{E}_{K} \cap \mathcal{E}_{\text {int }}$ and $\mathcal{E}_{K, \text { ext }}=\mathcal{E}_{K} \cap \mathcal{E}_{\text {ext }}$.

Definition 3.3 (Approximation space). Under hypothesis (1), let $\mathcal{D}=(\mathcal{M}, \mathcal{E}, \mathcal{P})$ be a a general FV discretization or a $\Delta$-adapted $F V$ discretization mesh of $\Omega$. The set $H_{\mathcal{D}}$ is defined as the set of functions from $\Omega$ to $\mathbb{R}$, constant on each element of $\mathcal{M}$.

\section{Approximation of the Dirichlet problem on $\Delta$-ADApted DISCRETIZATIONS}

4.1. Definition of the scheme. In this section, we consider the $\Delta$-adapted meshes of Definition 3.2 which allow a consistent discretization of the diffusion fluxes by a two point formula.

Remark 4.1 (Constraint on the point $x_{K}$ ). Definition 3.2 requires that $x_{K} \in K$. However, this condition is not always satisfied when using the well-known Delaunay triangulations. In fact, this condition is useless in the definition of the scheme itself. Furthermore, it also seems possible to relax it in the convergence analysis; however, it must then be replaced by some supplementary technical geometrical conditions, 
since, in particular, the property (5) is no longer satisfied. Additional complex developments are then required in the proofs, especially for the interpolation Lemma 4.4. Hence, for the sake of simplicity, we shall assume, as stated in Definition 3.2 that $x_{K} \in K$ holds for any $K \in \mathcal{M}$.

Definition 4.1 (Approximation space, interpolation operator, inner product and norm). Under assumptions (1), let $\mathcal{D}$ be an admissible FV discretization in the sense of Definition 3.2. To account for the homogeneous Dirichlet boundary conditions, we introduce the space

$$
H_{\mathcal{D}, 0}=\left\{u \in H_{\mathcal{D}}, u_{K}=0 \text { for all } K \in \mathcal{M} \text { such that } \mathcal{E}_{K, \text { ext }} \neq \emptyset\right\} .
$$

The interpolation operator $P_{\mathcal{D}}: C(\bar{\Omega}) \rightarrow H_{\mathcal{D}}, \varphi \mapsto P_{\mathcal{D}} \varphi$ is defined by:

$$
P_{\mathcal{D}} \varphi(x)=\varphi\left(x_{K}\right) \text { for a.e. } x \in K, \forall K \in \mathcal{M} \text {. }
$$

For any $u \in H_{\mathcal{D}}$, we introduce the jump of the function $u$ across an interface $\sigma$ with respect to the global orientation of the interfaces, and its local counterpart with respect to a cell $K \in \mathcal{M}$ :

$$
\delta_{\sigma} u=\left\{\begin{array}{r}
u_{K_{\sigma}^{+}}-u_{K_{\sigma}^{-}}, \forall \sigma \in \mathcal{E}_{\mathrm{int}} \\
0-u_{K_{\sigma}}, \forall \sigma \in \mathcal{E}_{\mathrm{ext}}
\end{array} \quad \text { and } \quad \delta_{K, \sigma} u=\left\{\begin{array}{r}
u_{L}-u_{K}\left(=\delta_{\sigma} u \boldsymbol{n}_{K, \sigma} \cdot \boldsymbol{n}_{\sigma}\right) \\
\forall \sigma=K \mid L \in \mathcal{E}_{K, \mathrm{int}} \\
-u_{K}\left(=\delta_{\sigma} u\right), \forall \sigma \in \mathcal{E}_{K, \mathrm{ext}} .
\end{array}\right.\right.
$$

The following symmetric bilinear form may be seen as a discrete equivalent of the inner product in $H_{0}^{1}(\Omega)$ :

$$
[u, v]_{\mathcal{D}}=\sum_{\sigma \in \mathcal{E}} \frac{|\sigma|}{d_{\sigma}} \delta_{\sigma} u \delta_{\sigma} v, \forall u, v \in\left(H_{\mathcal{D}}\right)^{2},
$$

and defines an inner product on $H_{\mathcal{D}}$. Moreover, the mapping $u \in H_{\mathcal{D}} \mapsto\|u\|_{\mathcal{D}}=$ $\left([u, u]_{\mathcal{D}}\right)^{1 / 2}$ defines a norm on $H_{\mathcal{D}, 0}$.

Recall that we have the property

$$
\left\|P_{\mathcal{D}} \varphi-\varphi\right\|_{L^{\infty}(\Omega)} \leq h_{\mathcal{D}}\|\nabla \varphi\|_{L^{\infty}(\Omega)}, \forall \varphi \in C^{1}(\bar{\Omega}) .
$$

The estimates and convergence analysis require a measure of the regularity of the mesh; we therefore define $\theta_{\mathcal{D}}$ by

$$
\theta_{\mathcal{D}}=\inf \left\{\frac{d_{K, \sigma}}{\operatorname{diam}(K)}, \frac{d_{K, \sigma}}{d_{\sigma}}, K \in \mathcal{M}, \sigma \in \mathcal{E}_{K}\right\} .
$$

We may now define the discrete operators which will be used in the discrete weak formulation.

Definition 4.2 (Discrete gradient and Laplace operators). Under Assumption (1), let $\mathcal{D}$ be a $\Delta$ adapted discretization of $\Omega$ in the sense of Definition [3.2, and let $u \in H_{\mathcal{D}}$. Then the discrete gradient $\nabla_{\mathcal{D}} u \in\left(H_{\mathcal{D}}\right)^{d}$ is defined by its constant values on the cells:

$$
\nabla_{K} u=\frac{1}{|K|} \sum_{\sigma \in \mathcal{E}_{K}} \frac{|\sigma|}{d_{\sigma}} \delta_{K, \sigma} u\left(x_{\sigma}-x_{K}\right)
$$


We also define a discrete Laplace operator $\Delta_{\mathcal{D}}: H_{\mathcal{D}} \rightarrow H_{\mathcal{D}}$ by its constant values on the primal cells:

$$
\Delta_{K} u=\frac{1}{|K|} \sum_{\sigma \in \mathcal{E}_{K}} \frac{|\sigma|}{d_{\sigma}} \delta_{K, \sigma} u, \forall K \in \mathcal{M}
$$

Note that

$$
-\int_{\Omega} u(x) \Delta_{\mathcal{D}} v(x) \mathrm{d} x=[u, v]_{\mathcal{D}}, \forall u, v \in\left(H_{\mathcal{D}}\right)^{2} .
$$

The discrete gradient $\nabla_{\mathcal{D}}$, defined by (16), is mimicking the consequence of the Stokes formula

$$
|K| \xi=\sum_{\sigma \in \mathcal{E}_{K}}|\sigma| \xi \cdot \boldsymbol{n}_{K, \sigma}\left(x_{\sigma}-x_{K}\right), \forall \xi \in \mathbb{R}^{d},
$$

which provides, in the case where $u(x)=\xi \cdot x$ and therefore $\nabla u=\xi$, then $\xi \cdot \boldsymbol{n}_{K, \sigma}=$ $\frac{\delta_{K, \sigma} u}{d_{\sigma}}$. It was first defined and shown to be consistent in 13. Definition 2.3, Lemma $2.5]$, in the sense that

$$
\left\|\nabla_{\mathcal{D}} P_{\mathcal{D}} \varphi-\nabla \varphi\right\|_{L^{2}(\Omega)^{d}} \leq C\left(\varphi, \theta_{\mathcal{D}}, \Omega\right) h_{\mathcal{D}}, \forall \varphi \in C^{2}(\bar{\Omega}),
$$

where $C\left(\varphi, \theta_{\mathcal{D}}, \Omega\right)$ only depends on $\varphi, \theta_{\mathcal{D}}$ and $\Omega$.

The discrete Laplace operator $\Delta_{\mathcal{D}}$, defined by (17), mimicks the finite volume formula

$$
\Delta_{K} u=\frac{1}{|K|} \int_{K} \Delta u(x) \mathrm{d} x=\frac{1}{|K|} \sum_{\sigma \in \mathcal{E}_{K}} \int_{\sigma} \nabla u(x) \cdot \boldsymbol{n}_{K, \sigma} \mathrm{d} s(x),
$$

and then we approximate $\nabla u(x) \cdot \boldsymbol{n}_{K, \sigma}$ by $\frac{\delta_{K, \sigma} u}{d_{\sigma}}$, thanks to the property that the straight line $\left(x_{K}, x_{L}\right)$ is orthogonal to $\sigma$.

We now approximate Problem (3) by

$$
\begin{aligned}
u \in H_{\mathcal{D}, 0}, \forall v \in H_{\mathcal{D}, 0}, \int_{\Omega} \Delta_{\mathcal{D}} u(x) \Delta_{\mathcal{D}} v(x) \mathrm{d} x \\
\quad=\int_{\Omega}\left(f(x) v(x)+\boldsymbol{g}(x) \cdot \nabla_{\mathcal{D}} v(x)+\ell(x) \Delta_{\mathcal{D}} v(x)\right) \mathrm{d} x .
\end{aligned}
$$

Remark 4.2. The term $\int_{\Omega} \boldsymbol{g}(x) \cdot \nabla v(x) \mathrm{d} x$ is approximated by $\int_{\Omega} \boldsymbol{g}(x) \cdot \nabla_{\mathcal{D}} v(x) \mathrm{d} x$ in (19). It could also be approximated by $\int_{\Omega} \boldsymbol{g}(x) \cdot \widetilde{\nabla}_{\mathcal{D}} v(x) \mathrm{d} x$, where the gradient $\widetilde{\nabla}_{\mathcal{D}}$ is piecewise constant on the diamond cells:

$$
\widetilde{\nabla}_{\mathcal{D}} u(x)=d \frac{\delta_{\sigma} u}{d_{\sigma}} \boldsymbol{n}_{\sigma}, \text { for a.e. } x \in D_{\sigma}, \forall \sigma \in \mathcal{E}, \forall u \in H_{\mathcal{D}} .
$$

It was first defined and proven to be weakly convergent in 11, Definition 2, Lemma 2]. We emphasize that it is not consistent, even in the case of rectangular meshes. Both options lead to a convergent scheme. The main difference is in the averaging formula for $\boldsymbol{g}$ (on the primal cells or on the diamond cells), and the choice is mainly decided by the data structure in the implementation of the scheme.

Remark 4.3. Considering the particular case $\boldsymbol{g}=0$ and $\ell=0$, we notice that (19) also reads

$$
u \in H_{\mathcal{D}, 0}, \forall v \in H_{\mathcal{D}, 0}, \sum_{K \in \mathcal{M}}|K| \Delta_{K} u \Delta_{K} v=\sum_{K \in \mathcal{M}} v_{K} \int_{K} f(x) \mathrm{d} x,
$$


and may therefore be interpreted as a finite volume scheme. Indeed, taking in (19), $v=1_{K}$ for $K \in \mathcal{M}$ with $\mathcal{E}_{K \text {,ext }}=\emptyset$, we obtain:

$$
\sum_{L \in \mathcal{M}}|L| \Delta_{L} u \Delta_{L} v=\sum_{\sigma \in \mathcal{E}_{K}} \frac{|\sigma|}{d_{\sigma}} \delta_{K, \sigma}\left(\Delta_{\mathcal{D}} u\right),
$$

which is a discrete equivalent of

$$
\int_{K} \Delta(\Delta u)(x) \mathrm{d} x=\sum_{\sigma \in \mathcal{E}_{K}} \int_{\sigma} \nabla(\Delta u)(x) \cdot \boldsymbol{n}_{K, \sigma} \mathrm{d} \gamma(x) .
$$

The scheme can then also be written as

$$
|K| \Delta_{K}\left(\Delta_{\mathcal{D}} u\right)=\int_{K} f(x) \mathrm{d} x, \forall K \in \mathcal{M} \text { such that } \mathcal{E}_{K, \text { ext }}=\emptyset
$$

and

$$
u_{K}=0, \forall K \in \mathcal{M} \text { such that } \mathcal{E}_{K, \text { ext }} \neq \emptyset .
$$

We can now derive the mathematical properties of the scheme, thanks to that of the discrete operator $\Delta_{\mathcal{D}}$, which has been the object of numerous studies (see e.g. [12]).

4.2. Study of the convergence of the scheme. We begin with some estimates on the approximate solutions. Because of the term $\int_{\Omega} \boldsymbol{g}(x) \cdot \nabla v(x) \mathrm{d} x$, we need the following stability result on the approximate gradient (see also [13]):

Lemma 4.1 (Stability of the discrete gradient). Let $\Omega$ be an open bounded connected polygonal subset of $\mathbb{R}^{d}, d \in \mathbb{N}^{\star}$, and let $\mathcal{D}$ be an admissible finite volume discretization of $\Omega$ in the sense of Definition 3.2 and let $0<\theta<\theta_{\mathcal{D}}$. Then

$$
\left\|\nabla_{\mathcal{D}} u\right\|_{L^{2}(\Omega)^{d}} \leq \frac{\sqrt{d}}{\theta_{\mathcal{D}}}\|u\|_{\mathcal{D}}, \forall u \in H_{\mathcal{D}} .
$$

Proof. By definition of the gradient (16), we have thanks to the Cauchy-Schwarz inequality and Definition (15):

$$
\begin{aligned}
\int_{\Omega}\left|\nabla_{\mathcal{D}} u\right|^{2} \mathrm{~d} x & =\sum_{K \in \mathcal{M}}|K|\left|\frac{1}{|K|} \sum_{\sigma \in \mathcal{E}_{K}} \frac{|\sigma|}{d_{\sigma}} \delta_{K, \sigma} u\left(x_{\sigma}-x_{K}\right)\right|^{2} \\
& \leq \sum_{K \in \mathcal{M}} \frac{1}{|K|} \sum_{\sigma \in \mathcal{E}_{K}}|\sigma| \frac{d_{K, \sigma}}{\theta} \sum_{\sigma \in \mathcal{E}_{K}}|\sigma| \frac{d_{K, \sigma}}{\theta}\left(\frac{\delta_{\sigma} u}{d_{\sigma}}\right)^{2} .
\end{aligned}
$$

The result follows from relation (5) and from the fact that

$$
\|u\|_{\mathcal{D}}^{2}=\sum_{K \in \mathcal{M}} \sum_{\sigma \in \mathcal{E}_{K}}|\sigma| d_{K, \sigma}\left(\frac{\delta_{\sigma} u}{d_{\sigma}}\right)^{2} .
$$

Lemma 4.2 (Existence, uniqueness and estimate on the solution of (19)). Let $\Omega$ be an open bounded connected polygonal subset of $\mathbb{R}^{d}, d \in \mathbb{N}^{\star}$, let $f \in L^{2}(\Omega)$, $\boldsymbol{g} \in L^{2}(\Omega)^{d}, \ell \in L^{2}(\Omega)$ and let $\mathcal{D}$ be an admissible finite volume discretization of $\Omega$ 
in the sense of Definition 3.2 and let $0<\theta<\theta_{\mathcal{D}}$. Then there exists $C>0$, only depending on $\Omega$ and $\theta$, such that for any $u \in H_{\mathcal{D}, 0}$ satisfying (19), then

$$
\begin{gathered}
\|u\|_{L^{2}(\Omega)} \leq C\left(\|f\|_{L^{2}(\Omega)}+\|\boldsymbol{g}\|_{L^{2}(\Omega)^{d}}+\|\ell\|_{L^{2}(\Omega)}\right), \\
\|u\|_{\mathcal{D}} \leq C\left(\|f\|_{L^{2}(\Omega)}+\|\boldsymbol{g}\|_{L^{2}(\Omega)^{d}}+\|\ell\|_{L^{2}(\Omega)}\right), \\
\left\|\nabla_{\mathcal{D}} u\right\|_{L^{2}(\Omega)^{d}} \leq C\left(\|f\|_{L^{2}(\Omega)}+\|\boldsymbol{g}\|_{L^{2}(\Omega)^{d}}+\|\ell\|_{L^{2}(\Omega)}\right),
\end{gathered}
$$

and

$$
\left\|\Delta_{\mathcal{D}} u\right\|_{L^{2}(\Omega)} \leq C\left(\|f\|_{L^{2}(\Omega)}+\|\boldsymbol{g}\|_{L^{2}(\Omega)^{d}}+\|\ell\|_{L^{2}(\Omega)}\right) .
$$

As a consequence, there exists one and only one $u \in H_{\mathcal{D}, 0}$ such that (19) holds.

Proof. We first recall the discrete Poincaré inequality [12]:

$$
\|v\|_{L^{2}(\Omega)} \leq \operatorname{diam}(\Omega)\|v\|_{\mathcal{D}}, \forall v \in H_{\mathcal{D}}
$$

therefore, thanks to (18) and (26), we get

$$
\|u\|_{\mathcal{D}} \leq \operatorname{diam}(\Omega)\left\|\Delta_{\mathcal{D}} u\right\|_{L^{2}(\Omega)} .
$$

Hence, setting $v=u$ in (19), and using the Cauchy-Schwarz inequality, we get

$$
\left\|\Delta_{\mathcal{D}} u\right\|_{L^{2}(\Omega)} \leq \operatorname{diam}(\Omega)^{2}\|f\|_{L^{2}(\Omega)}+\frac{\sqrt{d}}{\theta} \operatorname{diam}(\Omega)\|\boldsymbol{g}\|_{L^{2}(\Omega)^{d}}+\|\ell\|_{L^{2}(\Omega)},
$$

which proves

$$
\|u\|_{\mathcal{D}} \leq \operatorname{diam}(\Omega)\left(\operatorname{diam}(\Omega)^{2}\|f\|_{L^{2}(\Omega)}+\frac{\sqrt{d}}{\theta} \operatorname{diam}(\Omega)\|\boldsymbol{g}\|_{L^{2}(\Omega)^{d}}+\|\ell\|_{L^{2}(\Omega)}\right)
$$

and

$$
\|u\|_{L^{2}(\Omega)} \leq \operatorname{diam}(\Omega)^{2}\left(\operatorname{diam}(\Omega)^{2}\|f\|_{L^{2}(\Omega)}+\frac{\sqrt{d}}{\theta} \operatorname{diam}(\Omega)\|\boldsymbol{g}\|_{L^{2}(\Omega)^{d}}+\|\ell\|_{L^{2}(\Omega)}\right) .
$$

The three above inequalities provide (25), (23) and (22); note that the example provided in Section 6.1 indicates that the above inequalities lead to the optimal orders with respect to $\operatorname{diam}(\Omega)$. We then get (24) using (21). Finally, we conclude the existence and uniqueness of the solution to (19), which leads to a square linear system, from the estimate (22), setting $f=0, \boldsymbol{g}=0$ and $\ell=0$.

Lemma 4.3 (Compactness of a sequence of approximate solutions). Under assumption (1), let $\left(\mathcal{D}_{m}\right)_{m \in \mathbb{N}}$ be a sequence of $\Delta$-adapted discretizations (Definition 3.2) such that $h_{\mathcal{D}_{m}}$ tends to 0 as $m \rightarrow \infty$. Assume that there exists $\theta>0$ with $\theta<\theta_{\mathcal{D}_{m}}$ for all $m \in \mathbb{N}$. Let $\left(u_{m}\right)_{m \in \mathbb{N}}$ be a sequence of functions of $L^{2}(\Omega)$ satisfying $u_{m} \in H_{\mathcal{D}_{m}, 0}$ for all $m \in \mathbb{N}$. For simplicity, we shall denote the discrete operators $\nabla_{\mathcal{D}_{m}}$ and $\Delta_{\mathcal{D}_{m}}$ by $\nabla_{m}$ and $\Delta_{m}$, respectively. Assume that the sequence $\left(\Delta_{m} u_{m}\right)_{m \in \mathbb{N}}$ is bounded in $L^{2}(\Omega)$; then there exists a subsequence of $\left(\mathcal{D}_{m}\right)_{m \in \mathbb{N}}$, again denoted $\left(\mathcal{D}_{m}\right)_{m \in \mathbb{N}}$, and $u \in H_{0}^{2}(\Omega)$, such that the corresponding subsequence $\left(u_{m}\right)_{m \in \mathbb{N}}$ satisfies:

(1) $u_{m} \rightarrow u$ in $L^{2}(\Omega)$,

(2) $\nabla_{m} u_{m} \rightarrow \nabla u$ in $L^{2}(\Omega)^{d}$,

(3) $\Delta_{m} u_{m} \rightarrow \Delta u$ weakly in $L^{2}(\Omega)$,

as $m \rightarrow \infty$. 
Proof. Since the sequence $\left(\Delta_{m} u_{m}\right)_{m \in \mathbb{N}}$ is bounded in $L^{2}(\Omega)$, we may extract a subsequence of $\left(\mathcal{D}_{m}\right)_{m \in \mathbb{N}}$, such that $\left(\Delta_{m} u_{m}\right)_{m \in \mathbb{N}}$ converges weakly in $L^{2}(\Omega)$ to some $w \in L^{2}(\Omega)$. Since $\Delta_{m} u_{m}$ is bounded in $L^{2}(\Omega)$, we get that the same property holds for $\left\|u_{m}\right\|_{\mathcal{D}}$. Up to the extraction of a subsequence, we get from Lemma 5.7 of [15] that $u_{m}$ converges in $L^{2}(\Omega)$ to some function $u \in H_{0}^{1}(\Omega)$. First taking $v \in C_{c}^{\infty}(\Omega)$, we get

$$
\left[u_{m}, P_{\mathcal{D}_{m}} v\right]_{\mathcal{D}_{m}}=-\int_{\Omega} \Delta_{m} u_{m}(x) v(x) \mathrm{d} x .
$$

Passing to the limit $m \rightarrow \infty$ in the above relation and using Lemma 2.1 of [13], we get by density of $C_{c}^{\infty}(\Omega)$ in $H_{0}^{1}(\Omega)$ that

$$
\forall v \in H_{0}^{1}(\Omega), \int_{\Omega} \nabla u(x) \cdot \nabla v(x) \mathrm{d} x=-\int_{\Omega} w(x) v(x) \mathrm{d} x .
$$

It shows, by uniqueness of the limit $u$, that all the sequence $u_{m}$ corresponding to the extracted subsequence of $\left(\mathcal{D}_{m}\right)_{m \in \mathbb{N}}$ converges in $L^{2}(\Omega)$ to $u$. Let us denote by $\widetilde{u}_{m}$ the solution of the finite volume scheme

$$
\widetilde{u}_{m} \in H_{\mathcal{D}_{m}}, \quad-\Delta_{K} \widetilde{u}_{m}=\frac{1}{|K|} \int_{K} w(x) \mathrm{d} x, \forall K \in \mathcal{M}_{m} .
$$

Then, from Theorem 3.1 of [13], we get that $\left(\widetilde{u}_{m}\right)_{m \in \mathbb{N}}$ converges in $L^{2}(\Omega)$ to $u$ and $\left(\nabla_{m} \widetilde{u}_{m}\right)_{m \in \mathbb{N}}$ converges in $L^{2}(\Omega)^{d}$ to $\nabla u$. Let us observe that, using

$$
-\Delta_{K}\left(\widetilde{u}_{m}-u_{m}\right)=\frac{1}{|K|} \int_{K}\left(w(x)-\Delta_{m} u_{m}(x)\right) \mathrm{d} x,
$$

we have

$$
\left\|\widetilde{u}_{m}-u_{m}\right\|_{\mathcal{D}_{m}}^{2}=-\int_{\Omega}\left(w(x)-\Delta_{m} u_{m}(x)\right)\left(\widetilde{u}_{m}(x)-u_{m}(x)\right) \mathrm{d} x .
$$

We then get, using the strong convergence of $\widetilde{u}_{m}-u_{m}$ to 0 in $L^{2}(\Omega)$, that the right-hand side of the above equation tends to 0 . Hence $\left\|\widetilde{u}_{m}-u_{m}\right\|_{\mathcal{D}_{m}}$ tends to 0 as $m \rightarrow \infty$. Using (21) and the convergence of $\left(\nabla_{m} \widetilde{u}_{m}\right)_{m \in \mathbb{N}}$ in $L^{2}(\Omega)^{d}$ to $\nabla u$, we conclude that $\left(\nabla_{m} u_{m}\right)_{m \in \mathbb{N}}$ converges to $\nabla u$ in $L^{2}(\Omega)^{d}$. Since (28) holds, we have $\Delta u(x)=w(x)$ for a.e. $x \in \Omega$, which proves that $\Delta u \in L^{2}(\Omega)$. Let us prove that $u \in H_{0}^{2}(\Omega)$. Let $\bar{u}$ denote the prolongment of $u$ by 0 outside $\Omega$. Using the gradient defined by (20), we prolong it by $\widetilde{\nabla}_{\mathcal{D}_{m}} u_{m}$ by 0 in $\mathbb{R}^{d} \backslash \Omega$. Using the results of [1], we get that the sequence $\left(\widetilde{\nabla}_{\mathcal{D}_{m}} u_{m}\right)_{m \in \mathbb{N}}$ weakly converges to $\nabla \bar{u}$ in $L^{2}\left(\mathbb{R}^{d}\right)^{d}$.

Let $\varphi \in C_{c}^{\infty}\left(\mathbb{R}^{d}\right)$; note that $\varphi$ does not necessarily vanish at the boundary of $\Omega$. Hence we need to define

$$
\widehat{\delta}_{\sigma} \varphi=\varphi\left(x_{K_{\sigma}^{+}}\right)-\varphi\left(x_{K_{\sigma}^{-}}\right), \forall \sigma \in \mathcal{E}_{\text {int }} \text { and } \widehat{\delta}_{\sigma} \varphi=\varphi\left(z_{\sigma}\right)-\varphi\left(x_{K_{\sigma}}\right), \forall \sigma \in \mathcal{E}_{\text {ext }},
$$

where $z_{\sigma}$ is the orthogonal projection of $x_{K}$ on the hyperplane which contains $\sigma$. We then define a first order approximation $\mathbf{G}_{m} \varphi$ of $\nabla \varphi$ on $\mathbb{R}^{d}$ by

$\mathbf{G}_{m} \varphi(x)= \begin{cases}\frac{\widehat{\delta}_{\sigma} \varphi}{d_{\sigma}} \boldsymbol{n}_{\sigma}+\nabla \varphi(x)-\left(\nabla \varphi(x) \cdot \boldsymbol{n}_{\sigma}\right) \boldsymbol{n}_{\sigma}, & \text { for a.e. } x \in D_{\sigma}, \text { for all } \sigma \in \mathcal{E}, \\ \nabla \varphi(x), & \text { for a.e. } x \in \mathbb{R}^{d} \backslash \Omega .\end{cases}$ 
Let $T_{m}=\int_{\mathbb{R}^{d}} \widetilde{\nabla}_{m} u_{m}(x) \cdot \mathbf{G}_{m} \varphi(x) \mathrm{d} x$. Since $\widetilde{\nabla}_{m} u_{m}$ tends to $\nabla \bar{u}$ weakly in $L^{2}(\Omega)$ and $\mathbf{G}_{m} \varphi$ tends to $\nabla \varphi$ strongly in $L^{2}(\Omega)$, we get

$$
\lim _{m \rightarrow \infty} T_{m}=\int_{\mathbb{R}^{d}} \nabla \bar{u}(x) \cdot \nabla \varphi(x) \mathrm{d} x .
$$

Using the fact that $u_{K}$ is equal to 0 for any $K$ neighbouring the boundary, so that $\delta_{\sigma} u_{m}=0$ for any $\sigma \in \mathcal{E}_{\text {ext }}$, we may write

$$
\begin{aligned}
T_{m} & =\sum_{\sigma \in \mathcal{E}} \frac{|\sigma|}{d_{\sigma}}\left(\sigma u_{m}\right) \widehat{\delta}_{\sigma} \varphi=\sum_{\sigma \in \mathcal{E}_{\mathrm{int}}} \frac{|\sigma|}{d_{\sigma}} \delta_{\sigma} u_{m}\left(\varphi\left(x_{L}\right)-\varphi\left(x_{K}\right)\right) \\
& =-\sum_{K \in \mathcal{M}}|K| \varphi\left(x_{K}\right) \sum_{\sigma \in \mathcal{E}_{K}} \frac{|\sigma|}{d_{\sigma}} \delta_{\sigma} u_{m}=-\sum_{K \in \mathcal{M}}|K| \varphi\left(x_{K}\right) \Delta_{K} u_{m} .
\end{aligned}
$$

Hence

$$
T_{m}=-\int_{\Omega} P_{\mathcal{D}_{m}} \varphi(x) \Delta_{\mathcal{D}_{m}} u_{m}(x) \mathrm{d} x .
$$

Passing to the limit and using (14), we get

$$
\int_{\mathbb{R}^{d}} \nabla \bar{u}(x) \cdot \nabla \varphi(x) \mathrm{d} x=-\int_{\Omega} \varphi(x) w(x) \mathrm{d} x .
$$

This proves that $\nabla \bar{u} \in H_{\mathrm{div}}\left(\mathbb{R}^{d}\right)$ and that $\Delta \bar{u}=w$ a.e. in $\Omega$ and $\Delta \bar{u}=0$ outside $\Omega$. Since $\bar{u} \in H^{1}\left(\mathbb{R}^{d}\right)$, this implies that $\bar{u} \in H^{2}\left(\mathbb{R}^{d}\right)$ (this also is a consequence of (4), which holds with $\Omega=\mathbb{R}^{d}$ ). Since $\nabla \bar{u}=0$ in $\mathbb{R}^{d} \backslash \Omega$, we get that the trace of $\nabla \bar{u}$ on $\partial \Omega$ is equal to 0 . Hence $u \in H_{0}^{2}(\Omega)$.

As we mentioned in the introduction, the finite volume convergence analysis of the bi-harmonic operator relies on the fact that the discrete Laplace operator of some interpolate of a regular test function $\varphi$ tends strongly to $\Delta \varphi$. In previous studies, we used the interpolation operator $P_{\mathcal{D}}$ defined in (11), since we only used it for $\varphi$ itself or with the discrete gradient $\nabla_{\mathcal{D}}$, which is consistent. Here, however, we need to deal with the Laplace operator, and unfortunately, $\Delta_{\mathcal{D}}\left(P_{\mathcal{D}} \varphi\right)$ does not, in general, tend strongly to $\Delta \varphi$. Let us consider the simple example of a 1D discretization of $\Omega=(0,1)$ by the control volumes $K=((k-1) /(2 n), k /(2 n))$ for a nonzero integer $n$ and $k=1, \ldots, 2 n$, with $x_{K}=(k-0.25) /(2 n)$ for $k=$ $1,3 \ldots, 2 n-1$ and $x_{K}=(k-0.5) /(2 n)$ for $k=2,4, \ldots, 2 n$. For $\varphi(x)=x(1-x)$, we have $\Delta \varphi(x)=-2$ for all $x \in \Omega$, whereas $\Delta_{K}\left(P_{\mathcal{D}} \varphi\right)=-3 / 2$ for $K$ defined by $k=2,4, \ldots, 2 n-2$ and $\Delta_{K}\left(P_{\mathcal{D}} \varphi\right)=-5 / 2$ for $K$ defined by $k=3,5, \ldots, 2 n-1$.

Lemma 4.4 (Interpolation of regular functions with compact support). Let $\Omega$ be an open bounded connected polygonal subset of $\mathbb{R}^{d}, d \in \mathbb{N}^{\star}$, let $\mathcal{D}$ be an admissible finite volume discretization of $\Omega$ in the sense of Definition 3.2 and let $\theta>0$ with $\theta<\theta_{\mathcal{D}}$. Let $\varphi \in C_{c}^{2}(\Omega)$ and let $a=\mathrm{d}(\operatorname{support}(\varphi), \partial \Omega)$. Then there exists $\widetilde{P}_{\mathcal{D}} \varphi \in$ $H_{\mathcal{D}, 0}$ and $C>0$ only depending on $\theta$ such that

$$
\begin{aligned}
& \left\|\widetilde{P}_{\mathcal{D}} \varphi-\varphi\right\|_{L^{2}(\Omega)} \leq C h_{\mathcal{D}} \frac{|\varphi|_{2}}{a^{2}}, \\
& \left\|\widetilde{P}_{\mathcal{D}} \varphi-P_{\mathcal{D}} \varphi\right\|_{\mathcal{D}} \leq C h_{\mathcal{D}} \frac{|\varphi|_{2}}{a^{2}},
\end{aligned}
$$


and

$$
\left\|\Delta_{\mathcal{D}} \widetilde{P}_{\mathcal{D}} \varphi-\bar{\Delta}_{\mathcal{D}} \varphi\right\|_{L^{2}(\Omega)} \leq C h_{\mathcal{D}} \frac{|\varphi|_{2}}{a^{2}}
$$

where $|\varphi|_{2}=\max _{i, j=1, d}\left\|\partial_{i j}^{2} \varphi\right\|_{L^{\infty}(\Omega)}$ and $\bar{\Delta}_{\mathcal{D}} \varphi$ is the piecewise constant function equal to $\bar{\Delta}_{K} \varphi:=\frac{1}{|K|} \int_{K} \Delta \varphi(x) \mathrm{d} x$ in each $K \in \mathcal{M}$.

Proof. Let $\rho \in C_{c}^{\infty}\left(\mathbb{R}^{d}, \mathbb{R}_{+}\right)$be the function defined by

$$
\rho(x)=\frac{\exp \left(-1 /\left(1-|x|^{2}\right)\right)}{\int_{B(0,1)} \exp \left(-1 /\left(1-|y|^{2}\right)\right) \mathrm{d} y}, \forall x \in B(0,1),
$$

and $\rho(x)=0$ for $x \notin B(0,1)$. Let $\psi$ (see Figure 3 ) be the function defined by

$$
\psi(y)=\int_{x \in \Omega, \mathrm{d}(x, \partial \Omega)>\frac{a}{2}}\left(\frac{4}{a}\right)^{d} \rho\left(\frac{4}{a}(y-x)\right) \mathrm{d} x, \forall y \in \Omega .
$$

Then $\psi \in C_{c}^{\infty}(\Omega), \psi(x) \in[0,1]$ for all $x \in \Omega, \psi(x)=0$ for all $x \in \Omega$ such that $\mathrm{d}(x, \partial \Omega)<\frac{a}{4}$ and $\psi(x)=1$ for all $x \in \Omega$ such that $\mathrm{d}(x, \partial \Omega)>\frac{3 a}{4}$. The idea of construction of $\widetilde{P}_{\mathcal{D}} \varphi$ is to consider the discrete solution of the Laplace problem with the right-hand side $-\Delta \varphi$; since $\widetilde{P}_{\mathcal{D}} \varphi$ must be equal to 0 on the boundary cells, we multiply this discrete solution by $\psi$. Then the proof mimics the identity $\Delta(\psi v)=v \Delta \psi+2 \nabla \psi \cdot \nabla v+\psi \Delta v$.

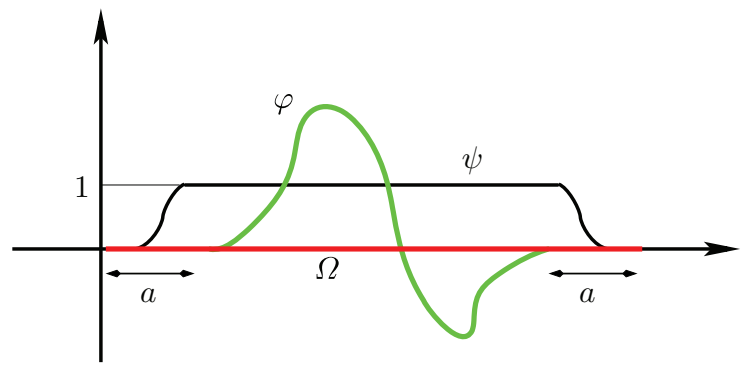

FiguRE 3. Functions $\varphi$ and $\psi$

We first suppose that $\mathcal{D}$ is such that $h_{\mathcal{D}}<\frac{a}{4}$. We denote in the following $\psi_{K}=\psi\left(x_{K}\right), \varphi_{K}=\varphi\left(x_{K}\right)$ for all $K \in \mathcal{M}$ and $\psi_{\mathcal{D}}=P_{\mathcal{D}} \psi, \varphi_{\mathcal{D}}=P_{\mathcal{D}} \varphi$. Let us define $\widetilde{v} \in H_{\mathcal{D}}$ such that

$$
-|K| \Delta_{K} \widetilde{v}=-\int_{K} \Delta \varphi(x) \mathrm{d} x, \forall K \in \mathcal{M},
$$

which is equivalent to

$$
\forall w \in H_{\mathcal{D}},[\widetilde{v}, w]_{\mathcal{D}}=-\int_{\Omega} \Delta \varphi(x) w(x) \mathrm{d} x .
$$

Let us remark that, thanks to (34), $\widetilde{v}$ satisfies

$$
|K| \psi_{K} \Delta_{K} \widetilde{v}=\int_{K} \Delta \varphi(x) \mathrm{d} x, \forall K \in \mathcal{M} .
$$

Indeed, if $\int_{K} \Delta \varphi(x) \mathrm{d} x \neq 0$, then $K \cap \operatorname{support}(\varphi) \neq \emptyset$, which implies $\mathrm{d}\left(x_{K}, \partial \Omega\right)>$ $\frac{3 a}{4}$, and therefore $\psi_{K}=1$. Otherwise, $\Delta_{K} \widetilde{v}=\int_{K} \Delta \varphi(x) \mathrm{d} x=0$. 
Using the results of [12, since the solution of the continuous Laplace problem is $\varphi \in C^{2}(\bar{\Omega})$, the following error estimates hold:

$$
\sum_{K \in \mathcal{M}}|K|\left(\widetilde{v}_{K}-\varphi_{K}\right)^{2} \leq C_{\Omega} h_{\mathcal{D}}^{2}|\varphi|_{2}^{2}
$$

and

$$
\sum_{\sigma \in \mathcal{E}} \frac{|\sigma|}{d_{\sigma}}\left(\delta_{\sigma}\left(\widetilde{v}-\varphi_{\mathcal{D}}\right)\right)^{2} \leq C_{\Omega} h_{\mathcal{D}}^{2}|\varphi|_{2}^{2}
$$

where $C_{\Omega}$ only depends on $\Omega$. We define $\widetilde{P}_{\mathcal{D}} \varphi \in H_{\mathcal{D}, 0}$ by its values in all $K \in \mathcal{M}$, given by $\widetilde{P}_{\mathcal{D}} \varphi_{K}=\psi_{K} \widetilde{v}_{K}$ (recall that, for all $K \in \mathcal{M}$ such that $\mathcal{E}_{K \text {,ext }} \neq \emptyset$, then $\mathrm{d}\left(x_{K}, \partial \Omega\right)<\frac{a}{4}$, hence $\left.\psi_{K}=0\right)$. We first remark that

$$
\left|\left(\widetilde{P}_{\mathcal{D}} \varphi\right)_{K}-\varphi_{K}\right|=\left|\psi_{K} \widetilde{v}_{K}-\psi_{K} \varphi_{K}\right| \leq\left|\widetilde{v}_{K}-\varphi_{K}\right|,
$$

which proves (30) thanks to (37) since $a \leq \operatorname{diam}(\Omega)$. Let us notice that the identity $a b-c d=c(b-d)+d(a-c)+(a-c)(b-d)$ yields

$$
\delta_{K, \sigma}\left(\widetilde{P}_{\mathcal{D}} \varphi\right)=\psi_{K} \delta_{K, \sigma} \widetilde{v}+\widetilde{v}_{K} \delta_{K, \sigma} \psi_{\mathcal{D}}+\delta_{K, \sigma} \widetilde{v} \delta_{K, \sigma} \psi_{\mathcal{D}}
$$

Hence we get

$$
|K| \Delta_{K}\left(\widetilde{P}_{\mathcal{D}} \varphi\right)=|K| \psi_{K} \Delta_{K} \widetilde{v}+|K| \widetilde{v}_{K} \Delta_{K} \psi_{\mathcal{D}}+\sum_{\sigma \in \mathcal{E}_{K}} \frac{|\sigma|}{d_{\sigma}} \delta_{K, \sigma} \psi_{\mathcal{D}} \delta_{K, \sigma} \widetilde{v}
$$

We remark that, for all $K \in \mathcal{M}$ such that $\Delta_{K} \psi_{\mathcal{D}} \neq 0$, then $\varphi_{K}=0$, and for all $\sigma \in \mathcal{E}$ such that $\delta_{\sigma} \psi_{\mathcal{D}} \neq 0$, then $\varphi_{K_{\sigma}^{+}}=\varphi_{K_{\sigma}^{-}}=0$. This leads, using (36), to

$$
|K| \Delta_{K}\left(\widetilde{P}_{\mathcal{D}} \varphi\right)=\int_{K} \Delta \varphi(x) \mathrm{d} x+|K|\left(\widetilde{v}_{K}-\varphi_{K}\right) \Delta_{K} \psi_{\mathcal{D}}+\sum_{\sigma \in \mathcal{E}_{K}} \frac{|\sigma|}{d_{\sigma}} \delta_{K, \sigma} \psi_{\mathcal{D}} \delta_{K, \sigma}\left(\widetilde{v}-\varphi_{\mathcal{D}}\right) .
$$

Moreover, a Taylor expansion provides

$$
\left|\delta_{K, \sigma} \psi_{\mathcal{D}}-d_{\sigma} \nabla \psi_{K} \cdot \boldsymbol{n}_{K, \sigma}\right| \leq d_{\sigma}^{2} \frac{C_{22}}{a^{2}},
$$

with $C_{22}$ is a constant. Since $\sum_{\sigma \in \mathcal{E}_{K}}|\sigma| \boldsymbol{n}_{K, \sigma}=0, \sum_{\sigma \in \mathcal{E}_{K}}|\sigma| d_{K, \sigma}=d|K|$ and $d_{\sigma} \leq d_{K, \sigma} / \theta$, we get

$$
|K|\left|\Delta_{K} \psi_{\mathcal{D}}\right| \leq \sum_{\sigma \in \mathcal{E}_{K}}|\sigma| d_{\sigma} \frac{C_{22}}{a^{2}} \leq \frac{C_{2}}{a^{2}}|K|,
$$

where $C_{2}$ only depends on $\theta$. Hence, using the notation $\bar{\Delta}_{K} \varphi=\frac{1}{|K|} \int_{K} \Delta \varphi(x) \mathrm{d} x$, we get

$$
\begin{aligned}
\sum_{K \in \mathcal{M}}|K|\left(\Delta_{K}\left(\widetilde{P}_{\mathcal{D}} \varphi\right)-\bar{\Delta}_{K} \varphi\right)^{2} \leq & 2 \frac{C_{2}^{2}}{a^{4}} \sum_{K \in \mathcal{M}}|K|\left(\widetilde{v}_{K}-\varphi_{K}\right)^{2} \\
& +2 \sum_{K \in \mathcal{M}} \frac{1}{|K|}\left(\sum_{\sigma \in \mathcal{E}_{K}} \frac{|\sigma|}{d_{\sigma}} \delta_{K, \sigma} \psi_{\mathcal{D}} \delta_{K, \sigma}\left(\widetilde{v}-\varphi_{\mathcal{D}}\right)\right)^{2}
\end{aligned}
$$


Thanks to the Cauchy-Schwarz inequality, we have

$$
\begin{aligned}
\left(\sum_{\sigma \in \mathcal{E}_{K}} \frac{|\sigma|}{d_{\sigma}} \delta_{K, \sigma} \psi_{\mathcal{D}} \delta_{K, \sigma}\left(\widetilde{v}-\varphi_{\mathcal{D}}\right)\right)^{2} & \leq \sum_{\sigma \in \mathcal{E}_{K}} \frac{|\sigma|}{d_{\sigma}}\left(\delta_{K, \sigma} \psi_{\mathcal{D}}\right)^{2} \sum_{\sigma \in \mathcal{E}_{K}} \frac{|\sigma|}{d_{\sigma}}\left(\delta_{K, \sigma}\left(\widetilde{v}-\varphi_{\mathcal{D}}\right)\right)^{2} \\
& \leq \frac{C_{1}^{2}}{a^{2}}|K| \sum_{\sigma \in \mathcal{E}_{K}} \frac{|\sigma|}{d_{\sigma}}\left(\delta_{K, \sigma}\left(\widetilde{v}-\varphi_{\mathcal{D}}\right)\right)^{2}
\end{aligned}
$$

where $C_{1}$ only depends on $\theta$. Hence

$$
\begin{aligned}
\sum_{K \in \mathcal{M}}|K|\left(\Delta_{K}\left(\widetilde{P}_{\mathcal{D}} \varphi\right)-\bar{\Delta}_{K} \varphi\right)^{2} \leq & 2 \frac{C_{2}^{2}}{a^{4}} \sum_{K \in \mathcal{M}}|K|\left(\widetilde{v}_{K}-\varphi_{K}\right)^{2} \\
& +2 \frac{C_{1}^{2}}{a^{2}} \sum_{K \in \mathcal{M}} \sum_{\sigma \in \mathcal{E}_{K}} \frac{|\sigma|}{d_{\sigma}}\left(\delta_{K, \sigma}\left(\widetilde{v}-\varphi_{\mathcal{D}}\right)\right)^{2} .
\end{aligned}
$$

This leads, thanks to (37) and (38), to

$$
\sum_{K \in \mathcal{M}}|K|\left(\Delta_{K}\left(\widetilde{P}_{\mathcal{D}} \varphi\right)-\bar{\Delta}_{K} \varphi\right)^{2} \leq \frac{2 C_{2}^{2}+4 C_{1}^{2} a^{2}}{a^{4}} C_{\Omega} h_{\mathcal{D}}^{2}|\varphi|_{2}^{2}
$$

Using the definition of $\bar{\Delta}_{\mathcal{D}} \varphi$, we get (32) thanks to $a \leq \operatorname{diam}(\Omega)$. Finally, since (39) can also be written

$$
\left\|\Delta_{\mathcal{D}}\left(\widetilde{P}_{\mathcal{D}} \varphi\right)-\Delta_{\mathcal{D}} \widetilde{v}\right\|_{L^{2}(\Omega)}^{2} \leq \frac{C}{a^{4}} h_{\mathcal{D}}^{2}|\varphi|_{2}^{2},
$$

we get, thanks to (18) and (26),

$$
\left\|\left(\widetilde{P}_{\mathcal{D}} \varphi\right)-\widetilde{v}\right\|_{\mathcal{D}}^{2} \leq \operatorname{diam}(\Omega)^{2} \frac{C}{a^{4}} h_{\mathcal{D}}^{2}|\varphi|_{2}^{2}
$$

Hence we deduce (31) from (38) using the triangle inequality and $a \leq \operatorname{diam}(\Omega)$.

In the case where $h_{\mathcal{D}} \geq \frac{a}{4}$, we set $\widetilde{P}_{\mathcal{D}} \varphi=0$. Since $\|\varphi\|_{L^{2}(\Omega)}$ and $\left\|\varphi_{\mathcal{D}}\right\|_{\mathcal{D}}$ are bounded, up to some constants only depending on $\Omega$, by $\|\Delta \varphi\|_{L^{2}(\Omega)}$, and using $\frac{1}{4} \leq \frac{h_{\mathcal{D}}}{a}$, we conclude that the lemma holds for all $h_{\mathcal{D}}>0$.

Remark 4.4. Lemma 4.4 is the main tool for a similar interpolation result which is needed in the convergence proof [10] for a finite volume discretization of the incompressible Navier-Stokes equations. In that case, we have to construct discrete test functions with homogeneous Dirichlet boundary values, which are discretely divergence-free and converge to regular divergence-free test functions with compact support.

Theorem 4.1 (Convergence of the scheme). Under assumptions (11)-(2), let $u \in$ $H_{0}^{2}(\Omega)$ be the solution of problem (3); let $\mathcal{D}$ be an $\Delta$-adapted $F V$ discretization of $\Omega$ in the sense of Definition 3.2 and $u_{\mathcal{D}} \in H_{\mathcal{D}, 0}$ be the solution of (19). Then, as $h_{\mathcal{D}}$ tends to 0 with $0<\theta \leq \theta_{\mathcal{D}}$ :

(1) $u_{\mathcal{D}}$ converges in $L^{2}(\Omega)$ to $u$,

(2) $\nabla_{\mathcal{D}} u_{\mathcal{D}}$ converges in $L^{2}(\Omega)^{d}$ to $\nabla u$,

(3) $\Delta_{\mathcal{D}} u_{\mathcal{D}}$ converges in $L^{2}(\Omega)$ to $\Delta u$.

Proof. Let $\left(\mathcal{D}_{m}\right)_{m \in \mathbb{N}}$ be a sequence of $\Delta$-adapted FV discretization of $\Omega$ in the sense of Definition 3.2 such that $h_{\mathcal{D}_{m}}$ tends to 0 as $m \rightarrow \infty$ and $\theta<\theta_{\mathcal{D}_{m}}$ for all $m \in \mathbb{N}$. Let $u_{m} \in H_{\mathcal{D}_{m}, 0}$, for all $m \in \mathbb{N}$, be the solution of (19). Thanks to Lemmas 4.2 and 4.3 . we get the existence of a subsequence of $\left(\mathcal{D}_{m}\right)_{m \in \mathbb{N}}$, again 
denoted $\left(\mathcal{D}_{m}\right)_{m \in \mathbb{N}}$, and of $u \in H_{0}^{2}(\Omega)$ such that the conclusion of Lemma 4.3 holds. Let $\varphi \in C_{c}^{\infty}(\Omega)$ be given. We take, in (19), $v=\tilde{P}_{m}(\varphi)$ where $\tilde{P}_{m}$ is defined by Lemma 4.4 for $\mathcal{D}=\mathcal{D}_{m}$. Passing to the limit in the scheme (thanks to weak/strong convergence) and by density of $C_{c}^{\infty}(\Omega)$ in $H_{0}^{2}(\Omega)$, we get that $u$ is the solution of problem (3). By a classical uniqueness argument, we get that the whole sequence converges. Setting $v=u_{m}$ in (19), we get the convergence of $\left\|\Delta_{\mathcal{D}_{m}} u_{m}\right\|_{L^{2}(\Omega)}^{2}$ to $\int_{\Omega}(f(x) u(x)+\boldsymbol{g}(x) \cdot \nabla u(x)+\ell(x) \Delta u(x)) \mathrm{d} x=\int_{\Omega}(\Delta u(x))^{2} \mathrm{~d} x$. Together with the weak convergence of $\Delta_{\mathcal{D}_{m}} u_{m}$ to $\Delta u$, this provides the convergence in $L^{2}(\Omega)$ of $\Delta_{\mathcal{D}_{m}} u_{m}$ to $\Delta u$.

Let us now state some error estimate results; for the sake of simplicity, we only prove them in the case $\boldsymbol{g}=0$ and $\ell=0$.

Theorem 4.2 (Error estimate in the case where $u \in C_{c}^{4}(\Omega)$ ). Let $\Omega$ be an open bounded connected polygonal subset of $\mathbb{R}^{d}, d \in \mathbb{N}^{\star}$. Let us assume that $u \in C_{c}^{4}(\Omega)$ is given and that $f=\Delta(\Delta u)$. Let $\mathcal{D}$ be an admissible finite volume discretization of $\Omega$ in the sense of Definition 3.2 and let $\theta>0$ with $\theta<\theta_{\mathcal{D}}$. Let $u_{\mathcal{D}} \in H_{\mathcal{D}, 0}$ be the solution of (19). Then there exists $C>0$, only depending on $\Omega, \theta$ and $u$ such that

$$
\begin{gathered}
\left\|u_{\mathcal{D}}-u\right\|_{L^{2}(\Omega)} \leq C h_{\mathcal{D}}, \\
\left\|\nabla_{\mathcal{D}} u_{\mathcal{D}}-\nabla u\right\|_{L^{2}(\Omega)^{d}} \leq C h_{\mathcal{D}},
\end{gathered}
$$

and

$$
\left\|\Delta_{\mathcal{D}} u_{\mathcal{D}}-\Delta u\right\|_{L^{2}(\Omega)} \leq C h_{\mathcal{D}}
$$

Remark 4.5. The above result is not optimal on regular grids, as shown in the numerical tests below, however, they seem to be optimal on some families of irregular grids.

Proof. In this proof, we denote by $C_{i}$ various positive quantities only depending on $\Omega, u$ and $\theta$. Let us first take any $w \in H_{\mathcal{D}, 0}$. We have

$$
\int_{\Omega} w(x) \Delta(\Delta u)(x) \mathrm{d} x=\int_{\Omega} w(x) f(x) \mathrm{d} x,
$$

which leads, thanks to $w_{K}=0$ if $K$ has a common boundary with $\partial \Omega$, to

$$
-\sum_{\sigma \in \mathcal{E}_{\text {int }}} \delta_{\sigma} w \int_{\sigma} \nabla(\Delta u)(x) \cdot \boldsymbol{n}_{\sigma} \mathrm{d} \gamma(x)=\sum_{K \in \mathcal{M}} w_{K} \int_{K} f(x) \mathrm{d} x .
$$

For $\sigma \in \mathcal{E}_{\text {int }}$, we set

$$
R_{\sigma}=\frac{1}{|\sigma|} \int_{\sigma} \nabla(\Delta u)(x) \cdot \boldsymbol{n}_{\sigma} \mathrm{d} \gamma(x)-\frac{\delta_{\sigma}\left(P_{\mathcal{D}} \Delta u\right)}{d_{\sigma}} .
$$

Thanks to the orthogonality property of the mesh, we have the existence of $C_{4}$, only depending on $u$, such that

$$
\left|R_{\sigma}\right| \leq C_{4} d_{\sigma}
$$

Using $w \in H_{\mathcal{D}, 0}$, we have

$$
\sum_{\sigma \in \mathcal{E}_{\text {int }}} \frac{|\sigma|}{d_{\sigma}} \delta_{\sigma} w \delta_{\sigma}\left(P_{\mathcal{D}} \Delta u\right)=\left[w, P_{\mathcal{D}} \Delta u\right]_{\mathcal{D}}
$$


Therefore, using (18), we have

$$
\sum_{K \in \mathcal{M}}|K| \Delta u\left(x_{K}\right) \Delta_{K} w=\sum_{K \in \mathcal{M}} w_{K} \int_{K} f(x) \mathrm{d} x+\sum_{\sigma \in \mathcal{E}_{\text {int }}}|\sigma| R_{\sigma} \delta_{\sigma} w .
$$

Let us now introduce some $v \in H_{\mathcal{D}, 0}$, which will be chosen later as some discrete interpolation of $u$. We have

$$
\begin{aligned}
\sum_{K \in \mathcal{M}}|K| \Delta_{K} v \Delta_{K} w= & \sum_{K \in \mathcal{M}} w_{K} \int_{K} f(x) \mathrm{d} x+\sum_{\sigma \in \mathcal{E}_{\text {int }}}|\sigma| R_{\sigma} \delta_{\sigma} w \\
& +\sum_{K \in \mathcal{M}}|K|\left(\Delta_{K} v-\Delta u\left(x_{K}\right)\right) \Delta_{K} w .
\end{aligned}
$$

We now subtract the above equation with (19), in which we replace $v$ by $w$ and we get

$$
\sum_{K \in \mathcal{M}}|K| \Delta_{\mathcal{D}}\left(v-u_{\mathcal{D}}\right) \Delta_{K} w=\sum_{\sigma \in \mathcal{E}_{\text {int }}}|\sigma| R_{\sigma} \delta_{\sigma} w+\sum_{K \in \mathcal{M}}|K|\left(\Delta_{K} v-\Delta u\left(x_{K}\right)\right) \Delta_{K} w
$$

Thanks to the Cauchy-Schwarz inequality, we have the existence of $C_{5}$ such that

$$
\left|\sum_{\sigma \in \mathcal{E}_{\text {int }}}\right| \sigma\left|R_{\sigma} \delta_{\sigma} w\right| \leq C_{5} h_{\mathcal{D}}\|w\|_{\mathcal{D}}
$$

which provides, thanks to (18), (26) and (27)

$$
\left|\sum_{\sigma \in \mathcal{E}_{\text {int }}}\right| \sigma\left|R_{\sigma} \delta_{\sigma} w\right| \leq C_{6} h_{\mathcal{D}}\left\|\Delta_{\mathcal{D}} w\right\|_{L^{2}(\Omega)} .
$$

Replacing $w$ by $\left(v-u_{\mathcal{D}}\right)$ we obtain

$$
\left\|\Delta_{\mathcal{D}}\left(v-u_{\mathcal{D}}\right)\right\|_{L^{2}(\Omega)} \leq C_{6} h_{\mathcal{D}}+\left(\sum_{K \in \mathcal{M}}|K|\left(\Delta_{K} v-\Delta u\left(x_{K}\right)\right)^{2}\right)^{\frac{1}{2}}
$$

Finally, we use the triangle inequality and obtain

$$
\begin{aligned}
\left\|\Delta u-\Delta_{\mathcal{D}} u_{\mathcal{D}}\right\|_{L^{2}(\Omega)} \leq & \left(\sum_{K \in \mathcal{M}} \int_{K}\left(\Delta u-\Delta u\left(x_{K}\right)\right)^{2} \mathrm{~d} x\right)^{\frac{1}{2}}+C_{6} h_{\mathcal{D}} \\
& +2\left(\sum_{K \in \mathcal{M}}|K|\left(\Delta_{K} v-\Delta u\left(x_{K}\right)\right)^{2}\right)^{\frac{1}{2}}
\end{aligned}
$$

Now we choose $v \in H_{\mathcal{D}, 0}$ according to Lemma 4.4 using $\varphi=u$. Thanks to (32) and $\Delta u \in C^{2}(\Omega)$, we get the existence of $C_{7}$ such that

$$
\left(\sum_{K \in \mathcal{M}}|K|\left(\Delta_{K} v-\Delta u\left(x_{K}\right)\right)^{2}\right)^{\frac{1}{2}} \leq C_{7} h_{\mathcal{D}}
$$

Gathering the above results, we get

$$
\left\|\Delta u-\Delta_{\mathcal{D}} u_{\mathcal{D}}\right\|_{L^{2}(\Omega)} \leq C_{8} h_{\mathcal{D}}
$$

and, thanks to (18) and (26),

$$
\left\|P_{\mathcal{D}} u-u_{\mathcal{D}}\right\|_{\mathcal{D}} \leq C_{9} h_{\mathcal{D}}
$$


and

$$
\left\|P_{\mathcal{D}} u-u_{\mathcal{D}}\right\|_{L^{2}(\Omega)} \leq C_{10} h_{\mathcal{D}}
$$

Using (21), we conclude the proof of the theorem.

Theorem 4.3 (Error estimate in the case where $u \in C^{4}(\bar{\Omega}) \cap H_{0}^{2}(\Omega)$ ). Let $\Omega$ be an open bounded connected polygonal subset of $\mathbb{R}^{d}, d \in \mathbb{N}^{\star}$. Let us assume that $u \in C^{4}(\bar{\Omega}) \cap H_{0}^{2}(\Omega)$ is given and that $f=\Delta(\Delta u)$. Let $\mathcal{D}$ be an admissible finite volume discretization of $\Omega$ in the sense of Definition 3.2 and let $\theta>0$ with $\theta<\theta_{\mathcal{D}}$. Let $u_{\mathcal{D}} \in H_{\mathcal{D}, 0}$ be the solution of (19). Then there exists $C>0$, only depending on $\Omega, \theta$ and $u$ such that

$$
\begin{gathered}
\left\|u_{\mathcal{D}}-u\right\|_{L^{2}(\Omega)} \leq C h_{\mathcal{D}}^{1 / 5}, \\
\left\|\nabla_{\mathcal{D}} u_{\mathcal{D}}-\nabla u\right\|_{L^{2}(\Omega)^{d}} \leq C h_{\mathcal{D}}^{1 / 5},
\end{gathered}
$$

and

$$
\left\|\Delta_{\mathcal{D}} u_{\mathcal{D}}-\Delta u\right\|_{L^{2}(\Omega)} \leq C h_{\mathcal{D}}^{1 / 5} .
$$

Remark 4.6. The above result is far from being optimal in the general case, this is due to the interpolation at the boundary.

Proof. For a given $a>0$ (which will be chosen later), we define the function $\psi_{a}$ by (33). We remark that the function $u_{a}$ defined by $u_{a}(x)=u(x) \psi_{a}(x)$ for all $x \in \Omega$ is such that

$$
\left\|\Delta u-\Delta u_{a}\right\|_{L^{2}(\Omega)} \leq C \sqrt{a},
$$

where $C$ only depends on $u$. Indeed, we have

$$
\Delta u_{a}(x)=\psi_{a}(x) \Delta u(x)+2 \nabla \psi_{a}(x) \cdot \nabla u(x)+u(x) \Delta \psi_{a}(x),
$$

which gives

$$
\Delta u_{a}(x)-\Delta u(x)=\left(\psi_{a}(x)-1\right) \Delta u(x)+2 \nabla \psi_{a}(x) \cdot \nabla u(x)+u(x) \Delta \psi_{a}(x) .
$$

Since there exists $C_{u}>0$, only depending on $u$, such that for all $x \in \Omega,|\nabla u(x)| \leq$ $C_{u} \mathrm{~d}(x, \partial \Omega)$ and $|u(x)| \leq C_{u} \mathrm{~d}(x, \partial \Omega)^{2}$, we get the existence of $C_{u}^{\prime}$, only depending on $u$, such that

$$
\left|\Delta u_{a}(x)-\Delta u(x)\right| \leq C_{u}^{\prime}, \forall x \in \Omega \text { such that } \mathrm{d}(x, \partial \Omega) \leq a,
$$

remarking that $\left|\nabla \psi_{a}(x)\right| \leq C_{0} / a$ and $\left|\Delta \psi_{a}(x)\right| \leq C_{0} / a^{2}$, with $C_{0}$ being a constant. Using $\Delta u_{a}(x)=\Delta u(x)$ if $\mathrm{d}(x, \partial \Omega)>a$, we get

$$
\left\|\Delta u-\Delta u_{a}\right\|_{L^{2}(\Omega)}^{2} \leq \operatorname{meas}(\partial \Omega) a\left(C_{u}^{\prime}\right)^{2} .
$$

We now reproduce the proof of Theorem 4.2 until the choice of $v \in H_{\mathcal{D}, 0}$, which is now given by Lemma 4.4 for $\varphi=u_{a}$. We then get that

$$
\sum_{K \in \mathcal{M}}|K|\left(\Delta_{K} v-\frac{1}{|K|} \int_{K} \Delta u_{a}(x) \mathrm{d} x\right)^{2} \leq C \frac{h_{\mathcal{D}}^{2}}{(a / 4)^{4}} .
$$

Using the triangle inequality we thus get the existence of $C_{11}$, only depending on $u$, such that

$$
\sum_{K \in \mathcal{M}}|K|\left(\Delta_{K} v-\Delta u\left(x_{K}\right)\right)^{2} \leq C_{11}\left(h_{\mathcal{D}}^{2}+a+\frac{h_{\mathcal{D}}^{2}}{a^{4}}\right) .
$$


It now suffices to choose $a=h_{\mathcal{D}}^{2 / 5}$ (note that, for small values of $h_{\mathcal{D}}$, then the case $h_{\mathcal{D}} \leq a / 4$ holds, which allows the function $v$ given by Lemma 4.4 to be different from 0 ), which leads to the conclusion of the proof.

\section{The CASE of General POlygonal Discretizations}

The scheme presented in Section 4 applies on admissible discretizations in the sense of Definition 3.2. which satisfy an orthogonality property restricting the type of meshes. In the present section, this scheme is generalized to general polygonal meshes in the sense of Definition [3.1, based on the SUCCES scheme presented in [1, 15, 14.

5.1. Definition of the scheme. Since we are now dealing with general meshes, we need more than two points to properly approximate the diffusion fluxes. The idea of 15 is to reconstruct a gradient from interface unknowns, stabilize it through the addition of a consistency error term (which vanishes as the mesh size tends to 0 ) and use it in a weak discrete form. When defined in this way, the scheme has a hybrid structure (SUSHI: Scheme Using Stabilization and Hybrid Interfaces), since it involves interface unknowns, whose related equations are the conservativity of the numerical fluxes. However, this scheme may be modified into a cell centered scheme by choosing the interface unknowns as combinations of the cell unknowns (and therefore relaxing the local conservativity of the numerical fluxes). The resulting scheme is compared to other cell centered schemes in [1] and applied to nonlinear problems in [14.

Definition 5.1 (Interface values). Let $\mathcal{D}=(\mathcal{M}, \mathcal{E}, \mathcal{P})$ be a $\mathrm{FV}$ mesh of $\Omega$ in the sense of Definition 3.1. For all $\sigma \in \mathcal{E}_{\text {int }}$ (where $\mathcal{E}_{\text {int }}$ denotes the set of interior faces and $\mathcal{E}_{\text {ext }}$ that of exterior faces), we choose a family of real numbers $\left(\beta_{\sigma}^{K}\right)_{K \in \mathcal{M}}$ (this family contains in general at most $d+1$ nonzero elements) such that:

$$
x_{\sigma}=\sum_{K \in \mathcal{M}} \beta_{\sigma}^{K} x_{K}, \text { with } \sum_{K \in \mathcal{M}} \beta_{\sigma}^{K}=1 .
$$

Then, for any $u \in H_{\mathcal{D}}$, and for any $\sigma \in \mathcal{E}_{\text {int }}$, we set

$$
u_{\sigma}=\sum_{K \in \mathcal{M}} \beta_{\sigma}^{K} u_{K}
$$

and for any $\sigma \in \mathcal{E}_{\text {ext }}$, we set

$$
u_{\sigma}=0 .
$$

Definition 5.2 (Discrete gradient). Under assumption (1), let $\mathcal{D}$ be a general FV discretization in the sense of Definition 3.1. For all $K \in \mathcal{M}$ and $\sigma \in \mathcal{E}_{K}$, we first define the following linear mappings:

$$
\begin{aligned}
\boldsymbol{G}_{K}: \mathbb{R}^{\mathcal{E}_{K}} \rightarrow \mathbb{R}^{d}, \quad \mathfrak{a} & =\left(a_{s}\right)_{s \in \mathcal{E}_{K}} \mapsto \boldsymbol{G}_{K}(\mathfrak{a})=\frac{1}{|K|} \sum_{s \in \mathcal{E}_{K}}|s| a_{s} \boldsymbol{n}_{K, s}, \\
R_{K, \sigma}: \mathbb{R}^{\mathcal{E}_{K}} \rightarrow \mathbb{R}, \quad \mathfrak{a} & =\left(a_{s}\right)_{s \in \mathcal{E}_{K}} \mapsto R_{K, \sigma}(\mathfrak{a}) \\
& =\frac{\sqrt{d}}{d_{K, \sigma}}\left(a_{\sigma}-\boldsymbol{G}_{K}(\mathfrak{a}) \cdot\left(x_{\sigma}-x_{K}\right)\right), \\
\boldsymbol{G}_{K, \sigma}: \mathbb{R}^{\mathcal{E}_{K}} \rightarrow \mathbb{R}^{d}, \quad \mathfrak{a} & =\left(a_{s}\right)_{s \in \mathcal{E}_{K}} \mapsto \boldsymbol{G}_{K, \sigma}(\mathfrak{a})=\boldsymbol{G}_{K}(\mathfrak{a})+R_{K, \sigma}(\mathfrak{a}) \boldsymbol{n}_{K, \sigma} .
\end{aligned}
$$


Note that if $\mathfrak{a}=\left(a_{s}\right)_{s \in \mathcal{E}_{K}}$ is such that $a_{s}=\boldsymbol{A} \cdot\left(x_{s}-x_{K}\right)$ for all $s \in \mathcal{E}_{K}$, with $\boldsymbol{A} \in \mathbb{R}^{d}$, then $\boldsymbol{G}_{K}(\mathfrak{a})=\boldsymbol{G}_{K, \sigma}(\mathfrak{a})=\boldsymbol{A}$ and $R_{K, \sigma}(\mathfrak{a})=0$. Using these linear mappings, we define

$$
\nabla_{K, \sigma} u=\boldsymbol{G}_{K, \sigma}\left(\mathfrak{d}_{K}(u)\right), \forall K \in \mathcal{M}, \forall \sigma \in \mathcal{E}_{K}, \forall u \in H_{\mathcal{D}},
$$

where $\mathfrak{d}_{K}(u)=\left(u_{s}-u_{K}\right)_{s \in \mathcal{E}_{K}} \in \mathbb{R}^{\mathcal{E}_{K}}$, and the values $\left(u_{s}\right)_{s \in \mathcal{E}}$ are defined in Definition 5.1 .

The discrete gradient $\nabla_{\mathcal{D}} u$ of $u \in H_{\mathcal{D}}$ is then defined as the piecewise constant function in each cone $D_{K, \sigma}$, for $K \in \mathcal{M}$ and $\sigma \in \mathcal{E}_{K}$ :

$$
\nabla_{\mathcal{D}} u(x)=\nabla_{K, \sigma} u, \forall x \in D_{K, \sigma}, \forall K \in \mathcal{M} \text { and } \sigma \in \mathcal{E}_{K} .
$$

Definition 5.3 (Discrete Laplace operator). Under assumption (1), let $\mathcal{D}$ be a general FV discretization in the sense of Definition 3.1. Let $u \in H_{\mathcal{D}}$, then $\Delta_{\mathcal{D}} u \in$ $H_{\mathcal{D}}$ is defined by its constant value $\Delta_{K} u$ on each cell $K$ of the mesh, given by:

$$
-\sum_{K \in \mathcal{M}}|K| v_{K} \Delta_{K} u=\int_{\Omega} \nabla_{\mathcal{D}} u(x) \cdot \nabla_{\mathcal{D}} v(x) \mathrm{d} x, \forall u, v \in H_{\mathcal{D}} .
$$

Note that (53) can be easily extended to more general second-order elliptic operators, as shown in 15 .

In order to account for the homogeneous Dirichlet boundary conditions, we introduce, for all $K \in \mathcal{M}$ and $\sigma \in \mathcal{E}_{K}$, the unique linear form $F_{K, \sigma}: \mathbb{R}^{\mathcal{E}_{K}} \rightarrow \mathbb{R}$ such that

$$
\begin{aligned}
\sum_{\sigma \in \mathcal{E}_{K}}\left|D_{K, \sigma}\right| \boldsymbol{G}_{K, \sigma}(\mathfrak{a}) \cdot \boldsymbol{G}_{K, \sigma}(\mathfrak{b})= & \sum_{\sigma \in \mathcal{E}_{K}} a_{\sigma} F_{K, \sigma}(\mathfrak{b}), \\
& \forall \mathfrak{a}=\left(a_{s}\right)_{s \in \mathcal{E}_{K}}, \mathfrak{b}=\left(b_{s}\right)_{s \in \mathcal{E}_{K}} \in \mathbb{R}^{\mathcal{E}_{K}} .
\end{aligned}
$$

Definition 5.4 (Discretization space). Under assumption (1), let $\mathcal{D}$ be a general FV discretization of $\Omega$ in the sense of Definition 3.1 For $u \in H_{\mathcal{D}}$ and $K \in \mathcal{M}$, let $\mathfrak{d}_{K}(u)=\left(u_{s}-u_{K}\right)_{s \in \mathcal{E}_{K}} \in \mathbb{R}^{\mathcal{E}_{K}}$, where the values $\left(u_{s}\right)_{s \in \mathcal{E}}$ are defined in Definition 5.1. The space $\tilde{H}_{\mathcal{D}, 0}$ is defined by

$$
\tilde{H}_{\mathcal{D}, 0}=\left\{u \in H_{\mathcal{D}} \text {, for all } \sigma \in \mathcal{E}_{\text {ext }}, F_{K_{\sigma}, \sigma}\left(\mathfrak{d}_{K_{\sigma}}(u)\right)=0\right\},
$$

where $K_{\sigma}$ denotes the unique control volume to which $\sigma$ is an interface.

Under assumption (2), we consider the following scheme for the discretization of (33) on a general discretization in the sense of Definition 3.1

Find $u \in \tilde{H}_{\mathcal{D}, 0}, \forall v \in \tilde{H}_{\mathcal{D}, 0}$,

$$
\int_{\Omega} \Delta_{\mathcal{D}} u(x) \Delta_{\mathcal{D}} v(x) \mathrm{d} x=\int_{\Omega}\left(f(x) P_{\mathcal{D}} v(x)+\boldsymbol{g}(x) \cdot \nabla_{\mathcal{D}} v(x)+\ell(x) \Delta_{\mathcal{D}} v(x)\right) \mathrm{d} x,
$$

Remark 5.1. We notice that, as in [15, the scheme (56) turns out to be identical to the scheme (19) on meshes such that $x_{\sigma}-x_{K}=d_{K, \sigma} \boldsymbol{n}_{K, \sigma}$, since, for all exterior face $\sigma$, the following relation holds:

$$
F_{K_{\sigma}, \sigma}\left(\mathfrak{d}_{K}(u)\right)=\frac{|\sigma|}{d_{K_{\sigma}, \sigma}}\left(0-u_{K_{\sigma}}\right) .
$$


5.2. Convergence analysis. As in Section 4 we need to measure some regularity of the mesh. We define:

$$
\begin{array}{r}
\theta_{\mathcal{D}}=\min \left\{\frac{h_{K}}{h_{M}}, \frac{h_{M}}{h_{K}}, \frac{d_{K, \sigma}}{h_{K}}, \frac{1}{\left|\beta_{\sigma}^{M}\right|}, \frac{h_{K}}{\left|x_{M}-x_{\sigma}\right|}, \frac{|\sigma|}{h_{K}^{d-1}},\right. \\
\left.K \in \mathcal{M}, \sigma \in \mathcal{E}_{K}, M \in \mathcal{M} \text { such that } \beta_{\sigma}^{M} \neq 0\right\} .
\end{array}
$$

Note that definition (57) implies a stronger regularity than that requested in [15] for the convergence of the scheme for second-order elliptic operators.

Lemma 5.1 (Existence, uniqueness and estimate on the solution of (56)). Under assumptions (11), (2), let $\mathcal{D}$ be a general $F V$ discretization of $\Omega$ in the sense of Definition 3.1 and let $0<\theta<\theta_{\mathcal{D}}$. Then there exists $C>0$, only depending on $\Omega$ and $\theta$, such that for any $u \in \tilde{H}_{\mathcal{D}, 0}$ satisfying (56), then

$$
\begin{gathered}
\|u\|_{L^{2}(\Omega)} \leq C\left(\|f\|_{L^{2}(\Omega)}+\|\boldsymbol{g}\|_{L^{2}(\Omega)^{d}}+\|\ell\|_{L^{2}(\Omega)}\right), \\
\left\|\nabla_{\mathcal{D}} u\right\|_{L^{2}(\Omega)^{d}} \leq C\left(\|f\|_{L^{2}(\Omega)}+\|\boldsymbol{g}\|_{L^{2}(\Omega)^{d}}+\|\ell\|_{L^{2}(\Omega)}\right) .
\end{gathered}
$$

and

$$
\left\|\Delta_{\mathcal{D}} u\right\|_{L^{2}(\Omega)} \leq C\left(\|f\|_{L^{2}(\Omega)}+\|\boldsymbol{g}\|_{L^{2}(\Omega)^{d}}+\|\ell\|_{L^{2}(\Omega)}\right) .
$$

As a consequence, there exists one and only one $u \in \tilde{H}_{\mathcal{D}, 0}$ such that (56) holds.

Proof. The proof is a straightforward consequence of the discrete definition (53) and of the discrete Poincaré inequality

$$
\|u\|_{L^{2}(\Omega)} \leq C\left\|\nabla_{\mathcal{D}} u\right\|_{L^{2}(\Omega)^{d}},
$$

provided in [15].

Lemma 5.2 (Compactness of a sequence of approximate solutions). Under assumptions (1), (2), let $\theta>0$ and let $\left(\mathcal{D}_{m}\right)_{m \in \mathbb{N}}$ be a sequence of general $F V$ discretizations $\Omega$ in the sense of Definition 3.1 such that $h_{\mathcal{D}_{m}}$ tends to 0 as $m \rightarrow \infty$ with $\theta<\theta_{\mathcal{D}_{m}}$ for all $m \in \mathbb{N}$. We assume that there exists $C>0$ and $u_{m} \in \tilde{H}_{\mathcal{D}_{m}, 0}$, for all $m \in \mathbb{N}$, such that (replacing the index $\mathcal{D}_{m}$ by $m$ for all discrete operators) $\left\|\Delta_{m} u_{m}\right\|_{L^{2}(\Omega)} \leq C$ for all $m \in \mathbb{N}$. Then there exists a subsequence of $\left(\mathcal{D}_{m}\right)_{m \in \mathbb{N}}$, again denoted $\left(\mathcal{D}_{m}\right)_{m \in \mathbb{N}}$, and $u \in H_{0}^{2}(\Omega)$, such that the corresponding subsequence $\left(u_{m}\right)_{m \in \mathbb{N}}$ satisfies

(1) the sequence $\left(u_{m}\right)_{m \in \mathbb{N}}$ converges in $L^{2}(\Omega)$ to $u$,

(2) the sequence $\left(\nabla_{m} u_{m}\right)_{m \in \mathbb{N}}$ converges in $L^{2}(\Omega)^{d}$ to $\nabla u$,

(3) the sequence $\left(\Delta_{m} u_{m}\right)_{m \in \mathbb{N}}$ weakly converges in $L^{2}(\Omega)$ to $\Delta u$.

Proof. The proof of Lemma 5.2 first uses the compactness arguments provided in 15]. In order to prove the $H_{0}^{2}(\Omega)$ regularity of the limit, one follows the same ideas as that of Lemma 4.3. Indeed, for a given $\varphi \in C_{c}^{\infty}\left(\mathbb{R}^{d}\right)$, we define $\varphi_{\sigma}=$ $\sum_{K \in \mathcal{M}} \beta_{\sigma}^{K} \varphi\left(x_{K}\right)$ for all $\sigma \in \mathcal{E}_{\text {int }}$ and $\varphi_{\sigma}=\varphi\left(x_{\sigma}\right)$ for all $\sigma \in \mathcal{E}_{\text {ext }}$. We finally define the function $\widehat{\nabla}_{\mathcal{D}} \varphi$ by the constant value $\boldsymbol{G}_{K, \sigma}\left(\widehat{\mathfrak{d}}_{K}(\varphi)\right)$ in $D_{K, \sigma}$, denoting $\widehat{\mathfrak{d}}_{K}(\varphi)=\left(\varphi_{\sigma}-\varphi\left(x_{K}\right)\right)_{\sigma \in \mathcal{E}_{K}}$. We then have

$$
-\sum_{K \in \mathcal{M}}|K| \varphi\left(x_{K}\right) \Delta_{K} u=\int_{\Omega} \nabla_{\mathcal{D}} u(x) \cdot \widehat{\nabla}_{\mathcal{D}} \varphi(x) \mathrm{d} x,
$$


since (54) and (55) imply that the values $\left(\varphi\left(x_{\sigma}\right)\right)_{\sigma \in \mathcal{E}_{\text {ext }}}$ at the boundary are multiplied by zero in the preceding relation, which then turns to be identical to (53). Thus, we again get the $H_{0}^{2}(\Omega)$ regularity of the limit in $L^{2}(\Omega)$ of $u_{m}$.

Lemma 5.3 (Interpolation of regular functions with compact support). Under assumptions (11), (2), let $\mathcal{D}$ be a general $F V$ discretization of $\Omega$ in the sense of Definition 3.1 and let $0<\theta<\theta_{\mathcal{D}}$. Let $\varphi \in C_{c}^{2}(\Omega)$ and let $a=\mathrm{d}(\operatorname{support}(\varphi), \partial \Omega)$. Then there exists $C>0$, only depending on $\theta$, and $\widehat{P}_{\mathcal{D}} \varphi \in \tilde{H}_{\mathcal{D}, 0}$ such that

$$
\begin{gathered}
\left\|\widehat{P}_{\mathcal{D}} \varphi-\varphi\right\|_{L^{2}(\Omega)} \leq C h_{\mathcal{D}} \frac{|\varphi|_{2}}{a^{2}}, \\
\left\|\nabla_{\mathcal{D}}\left(\widehat{P}_{\mathcal{D}} \varphi\right)-\nabla \varphi\right\|_{L^{2}(\Omega)^{d}} \leq C h_{\mathcal{D}} \frac{|\varphi|_{2}}{a^{2}},
\end{gathered}
$$

and

$$
\left\|\Delta_{\mathcal{D}}\left(\widehat{P}_{\mathcal{D}} \varphi\right)-\Delta \varphi\right\|_{L^{2}(\Omega)} \leq C h_{\mathcal{D}} \frac{|\varphi|_{2}}{a^{2}}
$$

where $|\varphi|_{2}=\max _{i, j=1, d}\left\|\partial_{i j}^{2} \varphi\right\|_{L^{\infty}(\Omega)}$.

Proof. Remarking that the conditions $F_{K, \sigma}\left(\mathfrak{d}_{K}(u)\right)=0$ are local, the proof of this lemma again follows that of Lemma 4.4. For a given function $\varphi \in C_{c}^{\infty}(\Omega)$, we again define the function $\psi$ by (33), as in the proof of Lemma 4.4. We then consider a discretization $\mathcal{D}$ such that $h_{\mathcal{D}} \leq a /\left(4 \theta^{2}\right)$. Thanks to definition (53), we get that

$$
\Delta_{K} u=\sum_{L \in \mathcal{M}} \tau_{K, L}\left(u_{L}-u_{K}\right)-\sum_{\sigma \in \mathcal{E}_{L} \cap \mathcal{E}_{\text {ext }}} \tau_{K, L}^{\sigma} u_{L}, \forall K \in \mathcal{M}, \forall u \in H_{\mathcal{D}}
$$

where $\tau_{K, L} \neq 0$ or $\tau_{K, L}^{\sigma} \neq 0$ imply $d\left(x_{K}, x_{L}\right) \leq h_{K} / \theta^{2}$, and there exists $C_{0}$, only depending on $\theta$, such that $\left|\tau_{K, L}\right| \leq C_{0} h_{K}^{d-2}$ and $\left|\tau_{K, L}^{\sigma}\right| \leq C_{0} h_{K}^{d-2}$. Indeed, there exist $C_{i}, i \in \mathbb{N}$, only depending on $\theta$, such that $\left|A_{L}^{\sigma \tau}\right| \leq C_{1} h_{K}^{d-2}$ for any $L \in \mathcal{M}$ with $\beta_{\sigma}^{L} \neq 0$ for some $\sigma \in \mathcal{E}_{K}$, the number of $L \in \mathcal{M}$ such that $\tau_{K, L} \neq 0$ is lower than $C_{2}$. We then define $\widehat{v} \in H_{\mathcal{D}}$ such that

$$
|K| \Delta_{K} \widehat{v}=\int_{K} \Delta \varphi(x) \mathrm{d} x, \forall K \in \mathcal{M}
$$

We have, thanks to the results of [15] (we again let in the following $\psi_{K}=\psi\left(x_{K}\right)$, $\varphi_{K}=\varphi\left(x_{K}\right)$ for all $\left.K \in \mathcal{M}\right)$ :

$$
\sum_{K \in \mathcal{M}}|K|\left(\widehat{v}_{K}-\varphi_{K}\right)^{2} \leq C_{\Omega, \theta} h_{\mathcal{D}}^{2}|\varphi|_{2}^{2}
$$

and

$$
\left\|\nabla_{\mathcal{D}} \widehat{v}-\nabla_{\mathcal{D}} P_{\mathcal{D}} \varphi\right\|_{L^{2}(\Omega)^{d}}^{2} \leq C_{\Omega, \theta} h_{\mathcal{D}}^{2}|\varphi|_{2}^{2},
$$

where $C_{\Omega, \theta}$ only depends on $\Omega$ and $\theta$. We then define $\widehat{P}_{\mathcal{D}} \varphi \in \tilde{H}_{\mathcal{D}, 0}$ by $\left(\widehat{P}_{\mathcal{D}} \varphi\right)_{K}=$ $\widehat{v}_{K} \psi_{K}$. We have

$$
|K| \Delta_{K}\left(\widehat{P}_{\mathcal{D}} \varphi\right)=|K| \psi_{K} \Delta_{K} \widehat{v}+|K| \widehat{v}_{K} \Delta_{K} \psi_{\mathcal{D}}+\sum_{L \in \mathcal{M}} \tau_{K, L}\left(\widehat{v}_{L}-\widehat{v}_{K}\right)\left(\psi_{L}-\psi_{K}\right) .
$$


This leads, using (36), to

$$
\begin{aligned}
|K| \Delta_{K}\left(\widehat{P}_{\mathcal{D}} \varphi\right)= & \int_{K} \Delta \varphi(x) \mathrm{d} x+|K|\left(\widehat{v}_{K}-\varphi_{K}\right) \Delta_{K} \psi_{\mathcal{D}} \\
& +\sum_{L \in \mathcal{M}} \tau_{K, L}\left(\widetilde{v}_{L}-\varphi_{L}-\left(\widehat{v}_{K}-\varphi_{K}\right)\right)\left(\psi_{L}-\psi_{K}\right) .
\end{aligned}
$$

Moreover, a Taylor expansion and definition (57) provide

$$
|K|\left|\Delta_{K} \psi_{\mathcal{D}}\right| \leq \sum_{L \in \mathcal{M}} \tau_{K, L} h_{K}^{2} \frac{C_{22}}{a^{2}} \leq \frac{C_{2}}{a^{2}}|K|,
$$

where $C_{22}$ and $C_{2}$ only depends on $\theta$ Hence we get

$$
\begin{aligned}
\sum_{K \in \mathcal{M}}|K|\left(\Delta_{K}\left(\widehat{P}_{\mathcal{D}} \varphi\right)-\frac{1}{|K|} \int_{K} \Delta \varphi(x) \mathrm{d} x\right)^{2} \leq 2 \frac{C_{2}^{2}}{a^{4}} \sum_{K \in \mathcal{M}}|K|\left(\widehat{v}_{K}-\varphi_{K}\right)^{2} \\
+2 \sum_{K \in \mathcal{M}} \frac{1}{|K|}\left(\sum_{L \in \mathcal{M}} \tau_{K, L}\left(\widehat{v}_{L}-\varphi_{L}-\left(\widehat{v}_{K}-\varphi_{K}\right)\right)\left(\psi_{L}-\psi_{K}\right)\right)^{2} .
\end{aligned}
$$

Then we use the fact that there exists $C_{3}$ only depending on $\theta$ such that

$$
\sum_{K, L \in \mathcal{M}} \tau_{K, L}\left(\widehat{v}_{L}-\varphi_{L}-\left(\widehat{v}_{K}-\varphi_{K}\right)\right)^{2} \leq C_{3}\left\|\nabla_{\mathcal{D}} \widehat{v}-\nabla_{\mathcal{D}} P_{\mathcal{D}} \varphi\right\|_{L^{2}(\Omega)^{d}}^{2}
$$

to conclude the proof in the same way as in the proof of Lemma 4.4.

Finally, the following convergence theorem holds (its proof is similar to that of Theorem 4.1).

Theorem 5.1 (Convergence of the scheme). Under assumptions (11), (2), let $u \in H_{0}^{2}(\Omega)$ be the solution of problem (3). Let $\mathcal{D}$ be a general $F V$ discretization of $\Omega$ in the sense of Definition 3.1 and let $u_{\mathcal{D}} \in \tilde{H}_{\mathcal{D}, 0}$ be the solution of (56). Then, as $h_{\mathcal{D}}$ tends to 0 with $0<\theta \leq \theta_{\mathcal{D}}$ :

(1) $u_{\mathcal{D}}$ converges in $L^{2}(\Omega)$ to $u$,

(2) $\nabla_{\mathcal{D}} u_{\mathcal{D}}$ converges in $L^{2}(\Omega)^{d}$ to $\nabla u$,

(3) $\Delta_{\mathcal{D}} u_{\mathcal{D}}$ converges in $L^{2}(\Omega)$ to $\Delta u$.

Error estimates can then also be derived.

Remark 5.2 (Writing the BiLaplacian SUCCES code from the Laplace SUCCES code). The implementation of this scheme is relatively easy starting from the SUCCES implementation of the Laplace equation. In fact the matrix obtained for the SUCCES discretization of the biharmonic operator is, up to the boundary conditions, equal to $A \operatorname{diag}(1 /|K|) A^{t}$, where $A$ is the Laplace matrix (recall that $A^{t}=A$ when using the SUCCES scheme) and $\operatorname{diag}(1 /|K|)$ denotes the diagonal matrix with coefficients $1 /|K|$. However, the treatment of the boundary condition $F_{K_{\sigma}, \sigma}\left(\mathfrak{d}_{K_{\sigma}}(u)\right)=0$ is not easy to perform directly in the matrix, in particular, because the stencil required by this condition is difficult to obtain. We chose to associate some Lagrange multipliers to these conditions (so we have card $\left(\mathcal{E}_{\text {ext }}\right)$ multipliers). Indeed, the solution to (56) may also be found by the minimization of $\frac{1}{2} \int_{\Omega}\left(\Delta_{\mathcal{D}} u(x)\right)^{2} \mathrm{~d} x-\int_{\Omega}\left(f(x) P_{\mathcal{D}} u(x)+\boldsymbol{g}(x) \cdot \nabla_{\mathcal{D}} u(x)+\ell(x) \Delta_{\mathcal{D}} u(x)\right) \mathrm{d} x$ for all $u \in H_{\mathcal{D}}$ under the constraints $F_{K_{\sigma}, \sigma}\left(\mathfrak{d}_{K_{\sigma}}(u)\right)=0$ for all $\sigma \in \mathcal{E}_{\text {ext }}$. This is then solved in 
practice, using a Lagrangian formulation for this minimization problem. The resulting matrix is then of the form

$$
\left(\begin{array}{cc}
A \operatorname{diag}(1 /|K|) A^{t} & B \\
B^{t} & 0
\end{array}\right)
$$

where $B$ denotes the matrix associated to the Lagrange multipliers of the condition $F_{K_{\sigma}, \sigma}\left(\mathfrak{d}_{K_{\sigma}}(u)\right)=0$ for all $\sigma \in \mathcal{E}_{\text {ext }}$.

\section{NuMERICAL RESULTS}

We apply the scheme (19) for the approximation of one, two, and three dimensional examples. We also consider 2D examples of application for scheme (56). We recall that the discrete Laplace operator is not consistent in the case of triangular meshes or nonuniform rectangular meshes with nonconstant space steps. Nevertheless, the discrete Laplacian of the approximate solution of the scheme converges to the Laplacian of the exact solution, as we show below. In the tables below we use for the difference of the approximate solution $u_{\mathcal{D}} \in H_{\mathcal{D}, 0}$ and the exact solution $u \in H_{0}^{2}(\Omega)$ the following discrete norms defined by

$$
\begin{aligned}
& E_{0}=\left(\sum_{K \in \mathcal{M}}|K|\left(u_{K}-u\left(x_{K}\right)\right)^{2} / \sum_{K \in \mathcal{M}}|K| u\left(x_{K}\right)^{2}\right)^{1 / 2}, \\
& E_{1}=\left(\sum_{K \in \mathcal{M}}|K|\left|\nabla_{K} u_{\mathcal{D}}-\nabla u\left(x_{K}\right)\right|^{2} / \sum_{K \in \mathcal{M}}|K|\left|\nabla u\left(x_{K}\right)\right|^{2}\right)^{1 / 2},
\end{aligned}
$$

and

$$
E_{2}=\left(\sum_{K \in \mathcal{M}}|K|\left(\Delta_{K} u_{\mathcal{D}}-\Delta u\left(x_{K}\right)\right)^{2} / \sum_{K \in \mathcal{M}}|K|\left(\Delta u\left(x_{K}\right)\right)^{2}\right)^{1 / 2},
$$

and similar notations in the case of the scheme (56). We also present, in the column "Stencil", the ratio between the number of nonzero terms in the matrix and the number of unknowns, which provides an evaluation of the size of the stencil of the scheme.

6.1. A one-dimensional example. We approximate the problem

$$
\begin{gathered}
u^{(4)}(x)=-1, x \in[0, L], \\
u(0)=u(L)=u^{\prime}(0)=u^{\prime}(L)=0,
\end{gathered}
$$

which is the classical problem of the completely fixed beam, under uniform load, using the scheme (19). The analytical solution is given by

$$
u(x)=-\frac{(x(L-x))^{2}}{24} .
$$

The exact minimum value of $u$ is $-L^{4} /\left(2^{4} 24\right) \simeq-0.002604167 L^{4}$. 
TABLE 1. Convergence orders, in the case $L=1$

\begin{tabular}{|c|c|c|c|c|c|c|c|c|c|}
\hline$n$ & Stencil & $E_{0}$ & order & $E_{1}$ & order & $E_{2}$ & order & $u_{\min }$ & $u_{\max }$ \\
\hline 100 & 4.84 & $4.29 \mathrm{E}-4$ & - & $6.27 \mathrm{E}-4$ & - & $1.12 \mathrm{E}-4$ & - & -0.0026031 & 0 \\
\hline 200 & 4.92 & $1.07 \mathrm{E}-4$ & $\simeq 2$ & $1.57 \mathrm{E}-4$ & $\simeq 2$ & $2.80 \mathrm{E}-5$ & $\simeq 2$ & -0.0026039 & 0 \\
\hline 400 & 4.96 & $2.68 \mathrm{E}-5$ & $\simeq 2$ & $3.92 \mathrm{E}-5$ & $\simeq 2$ & $6.99 \mathrm{E}-6$ & $\simeq 2$ & -0.0026041 & 0 \\
\hline
\end{tabular}

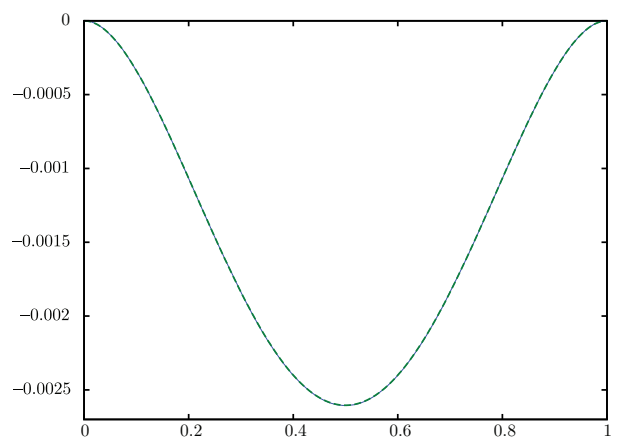

FiguRE 4. Exact and approximate solutions with $n=200$.

In this standard example, we get convergence with order 2 for $u, \nabla u$ and $\Delta u$. This convergence order is lower than that obtained using a conformal $H^{2}$ finite element method, but may be sufficient in practice. Since $f \equiv 1$, we have $\|f\|_{L^{2}(\Omega)}=$ $\sqrt{L}$; furthermore, $\|\Delta u\|_{L^{2}(\Omega)},\|\nabla u\|_{L^{2}(\Omega)}$ and $\|u\|_{L^{2}(\Omega)}$ are of the order $L^{n}\|f\|_{L^{2}(\Omega)}$ with, respectively, $n=2,3,4$. This shows that the constants found in the proof of Lemma 4.2 have the optimal order. The stencil size naturally tends to the 5-point stencil resulting from the "neighbours of the neighbours".

6.2. Two-dimensional examples. Here we compare the schemes (19) and (56) for the approximation of the $2 \mathrm{D}$ problem on $\Delta$-adapted grids, for which both schemes are defined. We choose the following function:

$$
u\left(x_{1}, x_{2}\right)=\left(1-\cos \left(2 \pi x_{1}\right)\right)\left(1-\cos \left(2 \pi x_{2}\right)\right), \forall\left(x_{1}, x_{2}\right) \in[0,1]^{2},
$$

which satisfies (3) for the ad hoc data $f=\Delta(\Delta u), \boldsymbol{g}=0, \ell=0, \Omega=] 0,1\left[{ }^{2}\right.$; hence we choose $f$ as the function defined by

$f\left(x_{1}, x_{2}\right)=\Delta(\Delta u)\left(x_{1}, x_{2}\right)=(2 \pi)^{4}\left(4 \cos \left(2 \pi x_{1}\right) \cos \left(2 \pi x_{2}\right)-\left(\cos \left(2 \pi x_{1}\right)+\cos \left(2 \pi x_{2}\right)\right)\right)$

Table 2 summarizes the results obtained for the scheme (19).

We see that again we get order 2 convergence for $u, \nabla u$ and $\Delta u$ for square meshes, but only order 1 convergence for triangular meshes. Again, this convergence order is lower than that obtained using a conformal $H^{2}$ finite element method; however, in $2 \mathrm{D}$ the conformal finite element methods are quite complex. For example, the Argyris triangular finite element is used to get a conformal approximation in $H^{2}(\Omega)$ requires 21 degrees of freedom [8] and the computation of the elementary rigidity matrix is much more complex than the present finite volume matrix. 
TABle 2. Convergence orders for the scheme (19) S: Squares meshes, T: Triangular meshes

\begin{tabular}{|c|c|c|c|c|c|c|c|c|c|}
\hline Mesh & Stencil & $E_{0}$ & order & $E_{1}$ & order & $E_{2}$ & order & $u_{\min }$ & $u_{\max }$ \\
\hline S 20x20 & 9.64 & $1.04 \mathrm{E}-2$ & - & $6.03 \mathrm{E}-3$ & - & $1.03 \mathrm{E}-2$ & - & 0 & 3.991 \\
\hline S 40x40 & 11.26 & $2.58 \mathrm{E}-3$ & $\simeq 2$ & $1.49 \mathrm{E}-3$ & $\simeq 2$ & $2.56 \mathrm{E}-3$ & $\simeq 2$ & 0 & 3.998 \\
\hline T 1400 & 9.10 & $3.99 \mathrm{E}-3$ & - & $5.27 \mathrm{E}-2$ & - & $5.97 \mathrm{E}-3$ & - & 0 & 3.998 \\
\hline T 5600 & 9.55 & $9.89 \mathrm{E}-4$ & $\simeq 2$ & $2.63 \mathrm{E}-2$ & $\simeq 1$ & $2.53 \mathrm{E}-3$ & $\geq 1$ & 0 & 3.9995 \\
\hline T 22400 & 9.77 & $2.47 \mathrm{E}-4$ & $\simeq 2$ & $1.31 \mathrm{E}-2$ & $\simeq 1$ & $1.20 \mathrm{E}-3$ & $\geq 1$ & 0 & 3.9999 \\
\hline
\end{tabular}

It is worth noticing that in the case of a triangular mesh, for any function $\varphi \in C_{c}^{\infty}(\Omega)$, the function $\Delta_{\mathcal{D}} P_{\mathcal{D}} \varphi$ never converges to $\Delta \varphi$ in $L^{2}(\Omega)$ when using triangular meshes, while the discrete Laplacian of the approximate solution tends to the Laplacian of the exact solution.

Note that the stencil tends to a 13-point stencil on structured square meshes, and to a 10-point stencil on triangular meshes (which are the number of "neighbours of neighbours").

Let us now compare the schemes (56) and (19). We first recall that if the points $x_{K}$ are chosen at the circumcenter point in (56) both schemes are identical on squares and triangles (see [15]), in which case the comparison is simple. However, if, in the case of the scheme (56), we let $x_{K}$ be the center of gravity of the triangles, the schemes are now different. We consider in Table 3 the same triangular meshes with such a discretization. We then remark that the order of convergence is reduced to 1 for $\nabla u$, a little more than 1 for $u$ but less than 1 for $\Delta u$. Moreover, the size of the matrices is very large (with a stencil size around 31 ). Hence, on $\Delta$-adapted meshes, the scheme (19) should be preferred to the scheme (56), since the results are more accurate for a much reduced computing price.

TABle 3. Convergence orders for the scheme (19) T: Triangular meshes. The meshes G1 and G2 are nonconforming quadrangular meshes depicted in Figure 6.

\begin{tabular}{|c|c|c|c|c|c|c|c|c|c|}
\hline Mesh & Stencil & $E_{0}$ & order & $E_{1}$ & order & $E_{2}$ & order & $u_{\min }$ & $u_{\max }$ \\
\hline T 1400 & 31.4 & $1.38 \mathrm{E}-3$ & - & $4.62 \mathrm{E}-2$ & - & $1.01 \mathrm{E}-2$ & - & $-3 . \mathrm{E}-4$ & 3.987 \\
\hline T 5600 & 31.2 & $3.88 \mathrm{E}-4$ & $\geq 1$ & $2.31 \mathrm{E}-2$ & $\simeq 1$ & $5.51 \mathrm{E}-3$ & $\leq 1$ & $-8 . \mathrm{E}-5$ & 3.9967 \\
\hline T 22400 & 31.1 & $1.42 \mathrm{E}-4$ & $\geq 1$ & $1.16 \mathrm{E}-2$ & $\simeq 1$ & $3.22 \mathrm{E}-3$ & $\leq 1$ & $-2 . \mathrm{E}-5$ & 3.9991 \\
\hline G1 1680 & 42.1 & $7.70 \mathrm{E}-3$ & - & $2.17 \mathrm{E}-2$ & - & $3.39 \mathrm{E}-2$ & - & -0.002 & 3.959 \\
\hline G2 6720 & 41.6 & $1.87 \mathrm{E}-3$ & $\simeq 2$ & $6.44 \mathrm{E}-3$ & $>1$ & $1.69 \mathrm{E}-2$ & $\simeq 1$ & -0.0006 & 3.990 \\
\hline
\end{tabular}

Let us now turn to the analysis of the results of the scheme (56) obtained on the same test case, but using meshes G1 and G2 depicted in Figure 6, note that on these meshes, the scheme (19) is no longer applicable. These meshes gather at least two difficulties: irregular control volumes and hanging nodes. We see that, even on these distorted meshes, the convergence rate remains quite good, but the computational cost, derived from the matrix size, increases (with a stencil size of around 42 terms). 


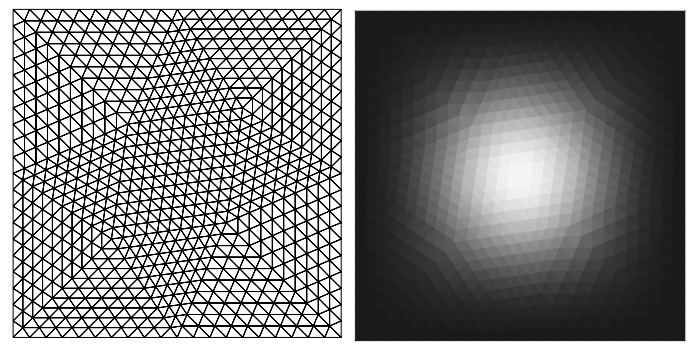

Figure 5. Grid T 1400 (left), solution with this grid (right).
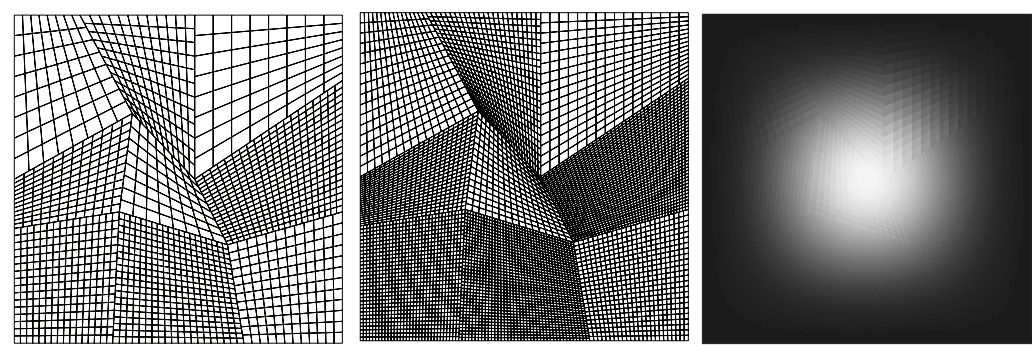

Figure 6. From left to right: grids G1, G2, solution with grid G2.

6.3. A three-dimensional example. We now use the scheme (19) for the approximation of the 3D problem, where the solution is given by (3) with $f=\Delta(\Delta u)$, $\boldsymbol{g}=0, l=0, \Omega=] 0,1\left[{ }^{3}\right.$ and

$u\left(x_{1}, x_{2}, x_{3}\right)=\left(1-\cos \left(2 \pi x_{1}\right)\right)\left(1-\cos \left(2 \pi x_{2}\right)\right)\left(1-\cos \left(2 \pi x_{3}\right)\right), \forall\left(x_{1}, x_{2}, x_{3}\right) \in[0,1]^{3}$.

We then have

$$
\begin{aligned}
\Delta(\Delta u)\left(x_{1}, x_{2}, x_{3}\right)=( & 2 \pi)^{4}\left(4 \left(\cos \left(2 \pi x_{1}\right) \cos \left(2 \pi x_{2}\right)+\cos \left(2 \pi x_{2}\right) \cos \left(2 \pi x_{3}\right)\right.\right. \\
& \left.+\cos \left(2 \pi x_{3}\right) \cos \left(2 \pi x_{1}\right)\right) \\
& -\left(\cos \left(2 \pi x_{1}\right)+\cos \left(2 \pi x_{2}\right)+\cos \left(2 \pi x_{3}\right)\right) \\
& \left.-9 \cos \left(2 \pi x_{1}\right) \cos \left(2 \pi x_{2}\right) \cos \left(2 \pi x_{3}\right)\right)
\end{aligned}
$$

We then have the following numerical results, for cubic meshes with $n^{3}$ control volumes.

TABle 4. Convergence orders

\begin{tabular}{|c|c|c|c|c|c|c|c|c|c|}
\hline Mesh & Stencil & $E_{0}$ & order & $E_{1}$ & order & $E_{2}$ & order & $u_{\min }$ & $u_{\max }$ \\
\hline $8 \times 8 \times 8$ & 7.7 & $0.721 \mathrm{E}-01$ & - & $0.564 \mathrm{E}-01$ & - & $7.49 \mathrm{E}-2$ & - & 0 & 7.57 \\
\hline $16 \times 16 \times 16$ & 14.8 & $0.175 \mathrm{E}-01$ & $\simeq 2$ & $0.134 \mathrm{E}-01$ & $\simeq 2$ & $1.82 \mathrm{E}-2$ & $\simeq 2$ & 0 & 7.90 \\
\hline $32 \times 32 \times 32$ & 19.4 & $0.435 \mathrm{E}-02$ & $\simeq 2$ & $0.329 \mathrm{E}-02$ & $\simeq 2$ & $4.52 \mathrm{E}-3$ & $\simeq 2$ & 0 & 7.98 \\
\hline 2000 Vor. & 39.3 & $0.958 \mathrm{E}-01$ & - & 0.238 & - & 0.281 & - & -0.015 & 7.83 \\
\hline 16000 Vor. & 59.8 & $0.475 \mathrm{E}-01$ & $\simeq 1$ & 0.114 & $\simeq 1$ & 0.172 & $\leq 1$ & -0.002 & 7.85 \\
\hline
\end{tabular}



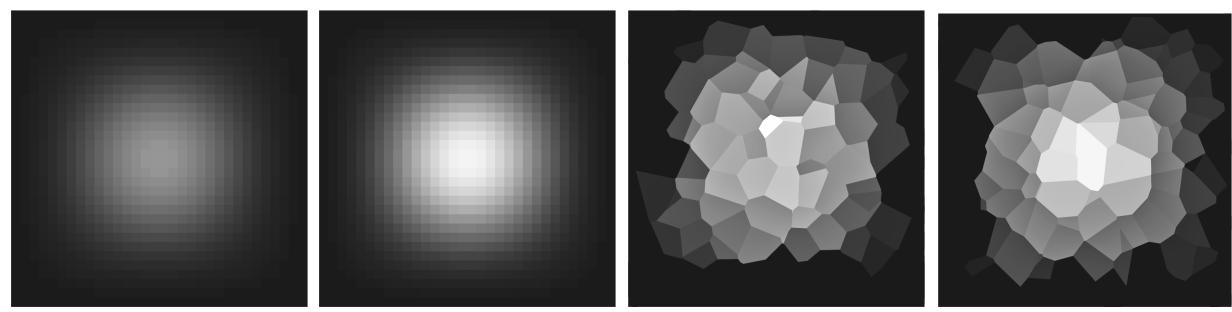

FiguRE 7. From left to right: solution obtained with mesh $32 \times 32 \times 32$, at $x_{1}=.2$, at $x_{1}=.5$, solution obtained with Voronoï mesh with 16000 control volumes, at $x_{1}=.2$ and at $x_{1}=.5$

In this $3 \mathrm{D}$ example, we again get convergence with order 2 for $u, \nabla u$ and $\Delta u$ with cubic meshes (the stencil tends to 25, which is the number of "neighbours of neighbours"). We recall that it is not possible to consider tetrahedral admissible meshes in 3D in the sense of Definition 3.2. The more general meshes that we can consider here are the Voronoi meshes (recall that the control volumes are defined, for any point $x_{K}$, as the set of the points of $\Omega$ closer to $x_{K}$ than to any point $x_{L}$ for $\left.L \neq K\right)$. Note that for such meshes there is no standard finite element technique available. In Table 4, we present the results obtained using two Voronoï meshes with, respectively, 2000 and 16000 control volumes. The centers of the control volumes are randomly generated. The convergence orders remain significant, although in this case again, for any function $\varphi \in C_{c}^{\infty}(\Omega)$, the function $\Delta_{\mathcal{D}} P_{\mathcal{D}} \varphi$ will not converge to $\Delta \varphi$ in $L^{2}(\Omega)$. We observe that the maximum and minimum values are not as precise as those obtained using cubic meshes. It is also interesting to notice that the resulting matrix is much sparser in comparison with cubic meshes, for comparable mesh sizes.

\section{Conclusion And Perspectives}

The finite volume schemes which are constructed in this paper for the discretization of the biharmonic problem were shown to be convergent and numerically efficient. Their convergence analysis relies on a discrete equivalent of the equality $\int_{\Omega} \nabla u \cdot \nabla v=-\int_{\Omega} u \Delta v$, together with a discrete Poincaré inequality, which allows the control of $\|\nabla u\|_{L^{2}(\Omega)^{d}}$ by $\|\Delta u\|_{L^{2}(\Omega)}$. A natural question is to recover such properties, starting from a Lagrange finite element interpolation. This demands that the discrete Laplacian be reconstructed thanks to the relation $\int_{\Omega} \nabla u \cdot \nabla v=-\int_{\Omega} u_{\mathcal{D}} \Delta_{\mathcal{D}} v$, for all pairs $u, v$ of elements of the finite element approximation space. This can easily be achieved, by associating a control volume with each interior degree of freedom, and by defining $u_{\mathcal{D}}$ as the piecewise constant function equal in this control volume to the corresponding degree of freedom. The difficulty is then to check that $\left\|u_{\mathcal{D}}-u\right\|_{L^{2}(\Omega)}$ can be controlled by $\|\nabla u\|_{L^{2}(\Omega)^{d}}$. This is easy to show in the case of triangles which are sufficiently acute, considering the "dual control volumes". To our knowledge, this property is open in the general case and could be the object of future works. 


\section{REFERENCES}

[1] L. Agelas, D. A. Di Pietro, and R. Masson. A symmetric and coercive finite volume scheme for multiphase porous media flow problems with applications in the oil industry. In Finite volumes for complex applications $V$, pages 35-51. ISTE, London, 2008.

[2] M. Ben-Artzi, I. Chorev, J.-P. Croisille, and D. Fishelov. A compact difference scheme for the biharmonic equation in planar irregular domains. SIAM J. Numer. Anal., 47(4):3087-3108, 2009. MR2551159(2010i:65235)

[3] M. Ben-Artzi, J.-P. Croisille, and D. Fishelov. A fast direct solver for the biharmonic problem in a rectangular grid. SIAM J. Sci. Comput., 31(1):303-333, 2008. MR2460780 (2009j:35066)

[4] C. Bi and L. Li. Mortar finite volume method with Adini element for biharmonic problem. J. Comput. Math., 22(3):475-488, 2004. MR2056302 (2005a:65126)

[5] S. C. Brenner and L.-Y. Sung. $C^{0}$ interior penalty methods for fourth order elliptic boundary value problems on polygonal domains. J. Sci. Comput., 22/23:83-118, 2005. MR 2142191 (2005m:65258)

[6] F. Brezzi and M. Fortin. Mixed and hybrid finite element methods. Springer-Verlag, New York, 1991. MR 1115205 (92d:65187)

[7] G. Chen, Z. Li, and P. Lin. A fast finite difference method for biharmonic equations on irregular domains and its application to an incompressible Stokes flow. Adv. Comput. Math., 29(2):113-133, 2008. MR2420868 (2009d:65145)

[8] P. Ciarlet. The finite element method. In P. G. Ciarlet and J.-L. Lions, editors, Part I, Handbook of Numerical Analysis, III. North-Holland, Amsterdam, 1991. MR1115235 (92f:65001)

[9] P. Destuynder and M. Salaün. Mathematical analysis of thin plate models. In Mathématiques E Applications [Mathematics \& Applications], volume 24. Springer-Verlag, Berlin, 1996. MR.1422248(2001e:74056)

[10] R. Eymard, J. Fuhrmann, and A. Linke. MAC schemes on triangular meshes. WIAS in: Finite Volumes for Complex Applications VI, Problems and Perspectives, Springer Proccedings in Mathematics, 2011, pp. 399-407.

[11] R. Eymard and T. Gallouët. H-convergence and numerical schemes for elliptic equations. SIAM Journal on Numerical Analysis, 41(2):539-562, 2003. MR2004187(2004h:65077)

[12] R. Eymard, T. Gallouët, and R. Herbin. Finite volume methods. In P. G. Ciarlet and J.-L. Lions, editors, Techniques of Scientific Computing, Part III, Handbook of Numerical Analysis, VII, pages 713-1020. North-Holland, Amsterdam, 2000. MR 1804748(2002e:65138)

[13] R. Eymard, T. Gallouët, and R. Herbin. A cell-centered finite-volume approximation for anisotropic diffusion operators on unstructured meshes in any space dimension. IMA J. Numer. Anal., 26(2):326-353, 2006. MR2218636(2007a:65170)

[14] R. Eymard, T. Gallouët, and R. Herbin. Cell centred discretization of fully nonlinear elliptic problems on general multidimensional polyhedral grids. J. Numer. Math., 17(3):173-193, 2009. MR2573566 (2010k:65230)

[15] R. Eymard, T. Gallouët, and R. Herbin. Discretisation of heterogeneous and anisotropic diffusion problems on general non-conforming meshes, sushi: a scheme using stabilisation and hybrid interfaces. IMA J. Numer. Anal., 2009. Advance Access published on September 7, 2009 IMAJNA, see also http://hal.archives-ouvertes.fr/. MR2727814

[16] R. Eymard, T. Gallouët, R. Herbin, and J.-C. Latché. Analysis tools for finite volume schemes. Acta Math. Univ. Comenian. (N.S.), 76(1):111-136, 2007. MR2331058(2008e:65243)

[17] T. Gallouët, R. Herbin, and M.-H. Vignal. Error estimates on the approximate finite volume solution of convection diffusion equations with general boundary conditions. SIAM J. Numer. Anal., 37(6):1935-1972 (electronic), 2000. MR1766855(2001e:65168)

[18] M. Gander and G. Wanner. From Euler, Ritz and Galerkin to modern computing. to appear in SIAM Review, 2011.

[19] E. H. Georgoulis and P. Houston. Discontinuous Galerkin methods for the biharmonic problem. IMA J. Numer. Anal., 29(3):573-594, 2009. MR2520159 (2010g:65209)

[20] T. Gudi, N. Nataraj, and A. K. Pani. Mixed discontinuous Galerkin finite element method for the biharmonic equation. J. Sci. Comput., 37(2):139-161, 2008. MR2453216 (2009j:65319)

[21] C. A. Hall, J. C. Cavendish, and W. H. Frey. The dual variable method for solving fluid flow difference equations on Delaunay triangulations. Comput. \& Fluids, 20(2):145-164, 1991. MR:1123813(92g:76059) 
[22] F. H. Harlow and J. E. Welch. Numerical calculation of time-dependent viscous incompressible flow of fluid with free surface. Physics of fluids, 8(12):2182-2189, 1965.

[23] I. Mozolevski, E. Süli, and P. R. Bösing. $h p$-version a priori error analysis of interior penalty discontinuous Galerkin finite element approximations to the biharmonic equation. J. Sci. Comput., 30(3):465-491, 2007. MR2295480(2008c:65341)

[24] J. Nicolaides, T. A. Porsching, and C. A. Hall. Covolume methods in computational fluid dynamics. In M. Hafez and K. Oshma, editors, Computation Fluid Dynamics Review, pages 279-299. John Wiley and Sons, New York, 1995.

[25] R. A. Nicolaides. Analysis and convergence of the MAC scheme. I. The linear problem. SIAM J. Numer. Anal., 29(6):1579-1591, 1992. MR1191137(93j:65143)

[26] S. V. Patankar. Numerical heat transfer and fluid flow. Series in Computational Methods in Mechanics and Thermal Sciences, Minkowycz and Sparrow Eds., 1980.

[27] W. Ritz. Über eine neue Methode zur Lösung gewisser Variationsprobleme der mathematischen Physik. Journal für die reine und angewandte Mathematik:111-114, 1857, Analysis and Applications, 135:1-61, 1908 .

[28] E. Süli and I. Mozolevski. $h p$-version interior penalty DGFEMs for the biharmonic equation. Comput. Methods Appl. Mech. Engrg., 196(13-16):1851-1863, 2007. MR2298696 (2008c:65350)

[29] T. Wang. A mixed finite volume element method based on rectangular mesh for biharmonic equations. J. Comput. Appl. Math., 172(1):117-130, 2004. MR2091134(2005h:65190)

Université Paris-Est Marne-la-Vallé́, Laboratoire D'Analyse et Mathématiques Appliquées, 5 boulevard Descartes, F-77454 Marne la Vallée Cedex 2

E-mail address: robert.eymard@univ-mlv.fr

Université Aix-Marseille, Laboratoire D'Analyse, Topologie et Probabilités, 39 rue Joliot-Curie, F-13453 Marseille Cedex France

E-mail address: thierry.gallouet@latp.univ-mrs.fr

Université Aix-Marseille, Laboratoire D'Analyse, Topologie et Probabilités, 39 rue Joliot-Curie, F-13453 MARseille CEDEX France

E-mail address: raphaele.herbin@latp.univ-mrs.fr

Weierstrass Institute, Mohrenstrasse 39, D-10117 Berlin, Germany

E-mail address: alexander.linke@wias-berlin.de 